\title{
Residence and redistribution of REE, Y, Zr, Th and U during granulite-facies metamorphism: behaviour of accessory and major phases in peraluminous granulites of central Spain
}

\author{
C. Villaseca ${ }^{\mathrm{a}, *}$, C. Martín Romera ${ }^{\mathrm{a}}, \mathrm{J}_{\text {. De la Rosa }}{ }^{\mathrm{b}}$, L. Barbero $^{\mathrm{c}}$ \\ ${ }^{a}$ Departamento Petrología y Geoquímica, Facultad Ciencias Geológicas, UCM, Universidad Complutense, 28040 Madrid, Spain \\ ${ }^{\mathrm{b}}$ Departamento Geología, Facultad CC. Experimentales, Campus El Carmen, 21071 Huelva, Spain \\ ${ }^{\mathrm{c}}$ Departamento Geología, Facultad Ciencias del Mar; UCA, 11510 Puerto Real, Cádiz, Spain
}

\begin{abstract}
Accessory minerals are thought to play a key role in controlling the behaviour of certain trace elements such as REE, Y, Zr, Th and U during crustal melting processes under high-grade metamorphic conditions. Although this is probably the case at middle crustal levels, when a comparison is made with granulite-facies lower crustal levels, differences are seen in trace element behaviour between accessory minerals and some major phases. Such a comparison can be made in Central Spain where two granulite-facies terranes have equilibrated under slightly different metamorphic conditions and where lower crustal xenoliths are also found. Differences in texture and chemical composition between accessory phases found in leucosomes and leucogranites and those of melanosomes and protholiths indicate that most of the accessory minerals in melt-rich migmatites are newly crystallized. This implies that an important redistribution of trace elements occurs during the early stages of granulite-facies metamorphism. In addition, the textural position of the accessory minerals with respect to the major phases is crucial in the redistribution of trace elements when melting proceeds via biotite dehydration melting reactions. In granulitic xenoliths from lower crustal levels, the situation seems to be different, as major minerals show high concentration of certain trace elements, the distribution of which is thus controlled by reactions involving final consumption of Al-Ti-phlogopite. A marked redistribution of HREE-Y $-\mathrm{Zr}$ between garnet and xenotime (where present) and zircon, but also of LREE between feldspars (K-feldspar and plagioclase) and monazite, is suggested.
\end{abstract}

Keywords: Accessory minerals; Trace elements; Granulite facies; Spain

\section{Introduction}

The trace element behaviour of accessory and major minerals during metamorphism is a topic re-

\footnotetext{
* Corresponding author. Fax: +34-915442535.

E-mail address: granito@geo.ucm.es (C. Villaseca).
}

cently investigated by a combination of microanalytical techniques (electron microprobe, LA-ICP-MS) and textural studies (abundance, position, zoning via $\mathrm{CL}, \mathrm{SEM}$ ), and deserves attention because of its consequences in metamorphism and crustal melting. Knowledge of trace element geochemistry of mineral phases is also crucial to understanding the behaviour 
-f REE-Y-Zr-Th-U during evelving metamorphic conditions and, especially, during crustal melting in high-temperature environments. The behaviour of accessory minerals and some other major minerals in granulite-facies conditions is pøorly constrained and this contrasts with studies of accessory minerals in felsic magmatic series (e.g. Gromet and Silver, 1983; Wark and Miller, 1993; Hoskin et al., 2000). In some granulite-terranes, an increase in growth of monazite and zircon with increasing temperature and metamorphism has been described (Franz et al., 1996; Schaltegger et al., 1999; Rubbatt et al., 2001). In -ther granulite terranes, accessory minerals in peraluminøus granulites have been consumed (xen॰time), or their population has dramatically decreased with metamorphic grade (e.g. Ivrea-Verban section, Bea and Mønter , 1999; or in løwer crustal xen॰liths, Reid, 1990). A change appears to occur in the growth of trace minerals during the amphibelite to granulite facies transition (Bingen et al., 1996). Three major factors may explain the behaviour of accessory minerals during high-grade metamorphism: (i) textural position, (ii) sølubility in melt, and (iii) behaviøur in metamorphic reactions.

This work presents a detailed study of the behaviøur of monazite, xenøtime and zircon from peraluminøus røcks (metapelites to felsic metaigne us lithøløgies) with increasing metamorphic grade. The samples form a middle-to-løwer crustal section (from migmatite terranes to lower crustal xen liths). We have investigated the combined textural and gechemical evolution of the main accessory minerals from well-characterize granulitic samples (Barber 1995 ; Barber et al., 1995; Villaseca et al., 1999, 2001; Martín Romera et al., 1999).

\section{Geological outline of the study area}

The migmatitic terranes of central Spain reached peak metamorphic conditions during the second Hercynian tectøn thermal stage $\left(\mathrm{M}_{2}\right)$ of low- $P$ /high- $T$ conditions following exhumation (Barber and Villaseca, 2000). Two areas are representative of these migmatitic terranes: an anatectic area in the Guadarrama mountains (Sotosalbos) and the Anatectic Com-

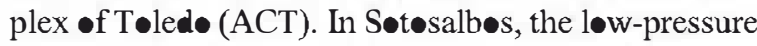
migmatites and related anatectic granites have cordi- erite and biotite as mafic minerals and their peak metamorphic conditions have been estimated to be around $750 \pm 50{ }^{\circ} \mathrm{C}$ and $4-5 \mathrm{~kb}$ (Martín Romera et al., 1999). The closely related Peña Negra anatectic area sh॰ws similar petrøgenetic characteristics (Bea et al., 1994; Pereira and Rodriguez Alonsø, 2000). In contrast, the ACT migmatites are characterized by gamet and cordierite as mafic minerals (biotite is more scarce) and their peak metamorphic conditions have been estimated to be around $800 \pm 25^{\circ} \mathrm{C}$ and $4-6 \mathrm{~kb}$ (Barbere, 1995).

The third sample of granulitic material is a suite of lower crustal xen-liths. Perrnian pest-Hercynian ultrabasic alkaline dykes carry a varied population of peraluminous granulitic xenoliths with mineral paragenesis indicative of equilibrium conditions (900$950^{\circ} \mathrm{C}$ and 9-11 kb) close to the base of the crust (Villaseca et al., 1999).

Although outcropping continuity between the groups of samples (migmatite terranes and xen-liths) is missing, all of the granulitic rocks originated during the same oregenical cycle and are composed of equivalent peraluminous lithøtypes, i.e., metasedimentary and felsic metaigneous rocks (Villaseca et al., 1999, 2001). Hence, an evaluation of the consequences of the increase in metamorphic conditions on accessory mineral assemblages can be established.

The age of granulitic metamørphism in this crustal section is unresølved. $\mathrm{U}-\mathrm{Pb}$ monazite ages from high-grade orthogneisses in the Guadarrama sector give $337 \pm 2 \mathrm{Ma}$ as a probable age of the $\mathrm{M}_{2}$ metamorphic peak (Escuder Viruete et al., 1998). In the Toledo sector, $\mathrm{U}-\mathrm{Pb}$ monazite ages from anatectic granitoids give a younger but more discordant value of $310 \pm 1 \mathrm{Ma}$ (Barber and Røgers, 1999). Møre speculative is the age of the granulitefacies metamorphism of the xenolith suite but their trapping by late-Hercynian alkaline lamprophyres $(283 \pm 30 \mathrm{Ma}$ in age, after Bea et al., 1999) has permitted a natural sampling of the Hercynian lower crust. Although there is an important diachronism in the age of high-grade metamorphism of the studied samples, the different granulitic assemblages presumably belong to the Hercynian metamorphic peak at different crustal levels, as this diachronism is similar to that obtained in tectonothermal models (e.g. Gerdes et al., 2000). 
3. Petrographic features of migmatites, granulites and related granites

A detaile petrographic description of the materials considered in this work can be found in several previous papers (Barber et al., 1995; Villaseca et al., 1998, 1999, 2001), thus, only a brief summary is given here.

\subsection{Sierra de Guadarrama migmatites and anatectic granites}

In the Sotosalbos area, cordierite-bearing granit ids are related to partially migmatized orth $\bullet$ neisses. These anatectic granitoids locally contain cordierite-rich nodules, corroded augen (megacrysts) -f K-feldspar, and orthøgneissic xenøliths, suggestive of their derivation from those felsic metaigne-us lithotypes (see alsø Martín Rømera et al., 1999). The Sotosalbos granitoid alsø shows sporadic mmscale biotite-rich enclaves that resemble regional melanısømes. Related cordierite-bearing migmatites usually display mafic nodules rimmed by leucocratic haløes that overprint the host-røck foliation. Søme migmatites are localized in narrow shear zones and provide evidence that melt segregation occurred. Typical mesøsøme-leucosøme pairs are rare as are mafic selvedges. In general, in situ residual granulitic material is difficult to find in the Guadarrama area.

Table 1 gives the modal composition (in v $\bullet . \%$ ) of the different units of this migmatitic area. The leucosomes consist dominantly of $\mathrm{K}$-feldspar with quartz, plagiøclase, minør biøtite and some cordierite or almandine-spessartine garnet (Table 1). Melanosomes have a more mafic character than the regional orthøgneisses, being rich in biotite, plagiøclase and quartz, and als• in accessery minerals. K-feldspar and cordierite are rare. The Sotosalbos granite is a medium-grained equigranular rock consisting of quartz, plagioclase, K-feldspar, biotite and cordierite with accessory tourmaline. Cordierite contains minor inclusiøns of biotite and sillimanite. Feldspar alsø contains needles of sillimanite. The scarce biotite-rich xenoliths resemble biotite-rich folia of the augen-gneisses. The most striking feature of these xenoliths is the high content of accessory minerals that are usually enclosed in biotite or plagioclase (Table 1).

\subsection{Anatectic com plex of Toledo}

In the Toledo Cømplex (ACT), the metasedimentary material mostly exhibits a stromatic-banded migmatitic structure. The melanosomes are mainly compose of cordierite, sillimanite, and garnet, with minor biotite, plagioclase, spinel and ilmenite (kinzigites) (Table 1). Well-developed coronas of cordierite appear around gamet. Cordierite commonly contains inclusiøns of quartz, sillimanite, biøtite, ilmenite, and spinel. Biotite is scarce and is usually interstitial to cordierite, suggesting that biotite dehydration melting reactions had almost nu to completion (Barberø, 1995). The leucosomes are characterized by a larger grain-size than the melanosømes. They are essentially composed of K-feldspar and quartz, with subordinate proportions of plagioclase (Table 1). The presence of large rounded gamet porphyroblasts (up to $7-8 \mathrm{~cm}$ ) with inclusions of sillimanite, biotite, and quartz is very characteristic. Accessory minerals are rare in

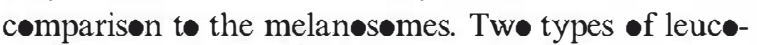
somes have been distinguished in this area on the basis of their gechemistry (Villaseca et al., 2001): (i) normal or N-type leucosomes with low-REE (and $\mathrm{Th}-\mathrm{Y}-\mathrm{Zr}$ ) contents and a positive chondrite-normalized Eu anomaly, and (ii) a high-REE leucosøme (enriched or E-type) with a negative Eu anomaly.

Related anatectic leucogranites (Cervatos-type) and restite-rich granitoids (Layos-type) appear in the ACT (Barber et al., 1995). The Layos granite is a suite ranging from quartz-rich tonalite to melamonzogranite, characterized by a high modal proportion of cordierite (up to $30 \%$ ) (Table 1). The Cervatos leucogranites $\bullet$ utcrops as vein-like or dyke-like concordant sheets, or eløngated massifs, in most cases associate with the Layos granites. These leucogranites have a modal eutectic composition and display centimetre-scale layering consisting of gamet-, cordierite- or very scarce biotite-bearing leucogranites. Essentially all of the mineral phases in both the anatectic granites and in the migmatitic granulites of the ACT have similar compositions (Barberø, 1995).

\subsection{Granulitic xenoliths from lower crustal levels and late Hercynian granites}

Three types of lower crustal granulites have been described by Villaseca et al. (1999) in the Sierra de 
Table 1

Granulite mineral assemblages

Modal analyses (vol\%) by point counting methods

\begin{tabular}{|c|c|c|c|c|c|c|c|c|c|c|c|c|c|c|c|}
\hline \multirow[b]{3}{*}{ Sample } & \multicolumn{7}{|c|}{ Anatectic Complex of Sotosalbos } & \multicolumn{5}{|c|}{ Anatectic Complex of Toledo } & \multicolumn{3}{|c|}{ Lower crustal xenoliths } \\
\hline & Orthogneiss & $\begin{array}{l}\text { Bt-rich } \\
\text { enclave }\end{array}$ & Melanosome & $\begin{array}{l}\text { Sotosalbos } \\
\text { granite }\end{array}$ & N-leucosome & N-leucosome & I eucogranite & Melanosome & $\begin{array}{l}\text { Layos } \\
\text { granite }\end{array}$ & N-leucosome & E-leucosome & $\begin{array}{l}\text { E-Cervatos } \\
\text { leucogranite }\end{array}$ & Pelitic & $\begin{array}{l}\text { Felsic } \\
\text { metaigneous }\end{array}$ & \\
\hline & 60870 & 100562 & 101637 & 100563 & 101638 & 102181 & 102183 & 93198 & 89103 & 9321 & 93197 & 81926 & $7775 \bullet$ & 99185 & \\
\hline Quartz & 26 & 1 & 17 & 32 & 19 & 29 & 20 & 18 & 22 & 24 & 22 & 34 & 21 & 28 & \\
\hline $\mathrm{K}$-feldspar & 27 & - & - & 19 & 46 & 52 & 49 & 27 & 15 & 60 & 59 & 27 & 33 & 24 & \\
\hline Plagioclase & 15 & 1.2 & 17 & 26 & 31 & 15 & 27 & 5 & 18 & 8 & 5 & 20 & - & 6 & \\
\hline Biotite & 25 & 73 & 42 & 10 & 3 & 1.5 & 3 & 21 & 19 & 0.8 & 3 & 3 & - & $\bullet .1$ & \\
\hline Gainet & 1 & - & - & - & - & $\operatorname{Tr}$ & - & 5 & - & 2 & 5 & 7 & 30 & 40 & \\
\hline Cordierite & $\operatorname{Tr}$ & - & 9.5 & 7 & $\operatorname{Tr}$ & - & $\operatorname{Tr}$ & 22 & 23 & 5 & 6 & 8 & - & - & \\
\hline Sillimanite & 6 & 21 & 12 & $\bullet .5$ & - & - & - & $\bullet .3$ & $\bullet .2$ & - & - & $\bullet .4$ & 12 & - & \\
\hline Opaques & $\operatorname{Tr}$ & 0.4 & 0.9 & 0.4 & - & - & $\operatorname{Tr}$ & 1.3 & $\bullet .2$ & $\bullet .2$ & 0.3 & 0.3 & 0.1 & 0.3 & \\
\hline Apatite & $\operatorname{Tr}$ & $\bullet .6$ & e. 6 & 0.4 & $\operatorname{Tr}$ & $\operatorname{Tr}$ & $\operatorname{Tr}$ & $\operatorname{Tr}$ & $\operatorname{Tr}$ & $\operatorname{Tr}$ & $\operatorname{Tr}$ & $\operatorname{Tr}$ & $\operatorname{Tr}$ & $\operatorname{Tr}$ & \\
\hline Other accs. & $\operatorname{Tr}$ & 1.1 & 0.3 & $\bullet .1$ & $\operatorname{Tr}$ & $\operatorname{Tr}$ & $\operatorname{Tr}$ & $\operatorname{Tr}$ & 0.1 & $\operatorname{Tr}$ & $\operatorname{Tr}$ & 0.3 & $\operatorname{Tr}$ & $\bullet .2$ & \\
\hline Tourmaline & & & & 3 & & & & & & & & & & & \\
\hline Rutile & & & & & & & & & & & & & 1.6 & 1.5 & \\
\hline Others & & Ms $(2 \%)$ & & Ms $(1.5 \%)$ & & Ms $(2 \%)$ & Ms $(1 \%)$ & Spl (Tr) & $\begin{array}{l}\text { Ms } \\
(3.7 \%)\end{array}$ & & & & & & \\
\hline \multicolumn{16}{|c|}{ Number of accessory crystals (per $\mathrm{cm}^{2}$ ) by SEM counting } \\
\hline & & & & 10056 & & & & & 93193 & & & & & 95148 & 99185 \\
\hline Apatite & 25 & 97 & 18 & 10 & 1 & & & 18 & 22 & 1 & 3 & & 1 & - & 2 \\
\hline Monazite & 55 & 164 & 68 & 16 & 8 & & & 48 & 53 & 4 & 29 & & 3 & 9 & - \\
\hline Zircon & 56 & 454 & 200 & 46 & 4 & & & $17 \bullet$ & 156 & 19 & 44 & & 17 & 55 & 21 \\
\hline Xenotime & 11 & 10 & - & 6 & 1 & & & 4 & 4 & - & - & & - & - & - \\
\hline
\end{tabular}


Guadarrama. They are rare felsic to intermediate chamockites ( $<0.01 \mathrm{v} \bullet 1 \%$ ), metapelites ( $5 \mathrm{v} \bullet 1 . \%$ ) and common felsic meta-igneous types (95 v॰l.\%). These granulites are granoblastic and many exhibit small-scale banding marked by gamet-rich bands alternating with felsic-rich layers, but clear migmatite structures are not evident. The felsic metaigneous granulites are quartzo-feldspathic garnetbearing types, sometimes with orthøpyroxene or sillimanite as accessories. The pelitic xenoliths have prismatic sillimanite as a majør mineral ( $>8$ v•l.\%) (Table 1).

The granulitic xenoliths are interpreted as the residual counterpart of the Hercynian granitic bathlith of the Spanish Central System (SCS) (Villaseca et al., 1999). The batholith is composed of dozens of plutons, mainly monzegranitic in modal composition, intruded during a time span of around $40 \mathrm{Ma}$ (Villaseca et al., 1998). These granites intrude and generate typical contact aure les in the exhumed middle-crust migmatite terranes previously described. A detailed study of the mineralogy, gechemistry and petrogenesis of these Hercynian peraluminous granites can be found in Villaseca et al. (1998) and Villaseca and Herreros (2000).

\section{Analytical methods}

Modes of the rocks from the migmatite terranes and xenøliths were derived from point counting of thin sections. Møre than 1200 points were counted in each $24 \times 46-\mathrm{mm}$ thin section. Because of the small size and difficulty in optical distinction, accessory mineral counting in a small area $\left(1 \mathrm{~cm}^{2}\right)$ was performed using a scanning electron microscope (SEM) (Table 1). Electrøn micrøprøbe analyses were carrie $\bullet$ ut $\bullet$ thin sectiøns at the Micrøscøía Electrónica CAI, University Complutense of Madrid. Before microchemical analyses, most of the thin sections were studied using a SEM equipped with an energy-dispersive spectrometric system. This methød unambiguously distinguished monazite from zircon and xenotime. Backscattered electron images were use as a guide during microprobe analysis. Some zircon and accessory concentrates from the three granulite sectors were als• •btained using standard heavy liquid and magnetic separation tech- niques, but most of the textural study was based on the thin sections.

Majør element concentrations of accessøry minerals (mønazite, xen॰time, zircon) were $\bullet$ btained by wavelength dispersive electrøn micrøpr be JEOL Superprobe JXA 8900-M equipped with four crystal spectrometers. Operating conditions were between 15 and $20 \mathrm{kV}$, and a beam current of approximately 20 to $50 \mathrm{nA}$ in spots of 2-5 $\mu \mathrm{m}$ in diameter. Abøut $24 \mathrm{~min}$ was necessary for each spot analysis. Absølute abundances for each element were determined by comparisøn with synthetic REE phosphates prepare by Jaresewich and Beatner (1991), and natural minerals for Zr, Y, U and Th. Error limits for each element depend strongly on the absolute concentration in each phase but are significant for the $<1$ wt.\% level (with error $>10 \%$ ). Concentrations below 0.2 wt.\% are merely qualitative.

Laser ablation-ICP-MS analyses of gamets and feldspars were obtained using a Hewlett Packard HP4500 Coupled Plasma-Mass Spectrometer (ICPMS) equipped with a CETAC LSX-1 00 laser ablation microprobe (LAM) at the University of Huelva. Operating conditions were optimised prior to analysis by using a 10-ppb solution of ${ }^{7} \mathrm{Li},{ }^{89} \mathrm{Y}$ and ${ }^{205} \mathrm{Tl}$. Plasma Ar fløw rate was $15.001 \mathrm{~min}^{-1}$; gas carrier and auxiliary flow rate were 1.25 and $0.51 \mathrm{~min}^{-1}$, respectively. Ablation craters were made using a Nd:YAG laser with 266-nm wavelength operating in Q-switch mode. The laser beam was optimised to produce ablation craters with diameters smaller than $70 \mu \mathrm{m}$. Selected isotopes were acquire by the TimeReselved Analysis (TRA) procedure, which allows the variation in concentration to be known at any time. In this way, the presence of either compositional zonation or mineral inclusions can be monitored (Longeritch et al., 1996). Corrections and final calculations of concentrations were made following Longeritch et al. (1996), using ${ }^{29} \mathrm{Si}$ as an intemal standard. Calibration was performed using the glass standard NIST RSM 612 which was analyzed four times before and two times after each run of 22 samples. The precision and accuracy were estimate using the ATHO-G standard, a MPI-DING reference glass (Jochurn et al., 2000). Precision is estimated to be between $2 \%$ and $10 \%$ for most of the analyzed trace elements. In general, an accuracy of $\pm 18 \%$ can be claimed for the LA-ICP-MS analyses, but agreement of measured 
Table 2

Sample suite accessory mineral assemblage

\begin{tabular}{|c|c|c|c|c|c|c|c|c|c|c|}
\hline Samples & Description & $\begin{array}{l}\text { Sample } \\
\text { suite }\end{array}$ & Monazite (mnz) & Zircon (zm) & Xenotime & $\mathrm{Ap}$ & Ilm & $\mathrm{Fe}-\mathrm{S}$ & Other & $\begin{array}{l}\text { Inclusions } \\
\text { in zircon }\end{array}$ \\
\hline $1000942-5$ & orthogneiss & Sotosalbos & $\begin{array}{l}\text { I-III? } \\
\text { (fels, bt, m) }\end{array}$ & B-C (bt, fels) & II (bt, qrz) & $\mathrm{X}$ & $\mathrm{X}$ & $\mathrm{X}$ & & \\
\hline $6087-62458$ & orthogneiss & Sotosalbos & I (bt, fels, m) & $\mathbf{B}-\mathrm{C} ?-\mathbf{D}(\mathrm{bt}, \mathrm{m})$ & $I$ (fels) & $\mathrm{X}$ & $\mathrm{X}$ & $\mathrm{X}$ & Gr & \\
\hline 102175 & melanosome & Sotosalbos & III (fels, m) & $\mathbf{B}(\mathrm{bt}, \mathrm{m})$ & & $\mathrm{X}$ & $\mathrm{X}$ & $\mathrm{X}$ & & $a p, b t$ \\
\hline 100562 & $\begin{array}{l}\text { bt-rich } \\
\text { enclave }\end{array}$ & Sotosalbos & I-III (bt, ap, m) & $\mathbf{B} ?-\mathrm{C}(\mathrm{bt}, \mathrm{m})$ & I (bt, m) & $\mathrm{X}$ & $\mathrm{X}$ & $\mathrm{X}$ & $\begin{array}{l}\mathrm{Si}-\mathrm{Y}-\mathrm{REE} \\
\text { carbonate? }\end{array}$ & \\
\hline 102183 & leucogranite & Sotosalbos & $\begin{array}{l}\text { II - III } \\
\text { (bt, fels, m) }\end{array}$ & $\mathrm{C}(\mathrm{bt}, \mathrm{m})$ & II (bt) & $\mathrm{X}$ & $\mathrm{X}$ & & & \\
\hline $100560-3$ & granitoid & Sotosalbos & II- III (fels, m) & $\begin{array}{l}\text { A ?-B-C } \\
\text { (bt, fels, m) }\end{array}$ & I & $\mathrm{X}$ & $\mathrm{X}$ & $\mathrm{X}$ & Cheralite & \\
\hline 93197 & leucosome & Toledo & I-III (fels, bt, m) & $\begin{array}{l}\text { C-D } \\
\text { (fels, bt, grt, m) }\end{array}$ & & $\mathrm{X}$ & $\mathrm{X}$ & $\mathrm{X}$ & & $\mathrm{mnz}$ \\
\hline $81925-6-87202$ & leucogranite & Toledo & I-III (fels, bt, m) & $\begin{array}{l}\mathbf{B}-\mathrm{C}-\mathbf{D} \\
\text { (fels, bt, m) }\end{array}$ & & $\mathrm{X}$ & $\mathrm{X}$ & $\mathrm{X}$ & & \\
\hline 93193 & $\begin{array}{l}\text { Layos } \\
\text { granitoid }\end{array}$ & Toledo & I-III (fels, bt, m) & $\begin{array}{l}\mathrm{A}-\mathbf{B}-\mathrm{C}-\mathbf{D} \\
(\mathrm{f} \text { els, bt, m) }\end{array}$ & $\mathrm{I}(\mathrm{m}, \mathrm{bt})$ & $\mathrm{X}$ & $\mathrm{X}$ & $\mathrm{X}$ & & $\mathrm{qt} 2$, sil \\
\hline $77750 / \mathrm{U}-46$ & pelitic & Xenoliths & $\begin{array}{l}\text { I-III } \\
(\mathrm{qt} 2, \text { fels, m) }\end{array}$ & $\begin{array}{l}\mathbf{B}-\mathrm{C}-\mathbf{D} \\
(\mathrm{qt} 2, \text { fels, sil, m) }\end{array}$ & & $\mathrm{X}$ & $\mathrm{X}$ & $\mathrm{X}$ & $\mathbf{R t}$, Gr & sil \\
\hline $81846-95151$ & felsic & Xenoliths & I-III (fels, m) & $\begin{array}{l}\text { A?-C-D } \\
\text { (fels, grt, m) }\end{array}$ & & $\mathrm{X}$ & $\mathrm{X}$ & $\mathrm{X}$ & $\mathbf{R t}$ & \\
\hline $95148-99185$ & felsic & Xenoliths & $\begin{array}{l}\text { III } \\
\text { (fels, grt, sil, m) }\end{array}$ & $\begin{array}{l}\mathbf{B}-\mathrm{C}-\mathbf{D} \\
\text { (fels, grt, sil, m) }\end{array}$ & & $\mathrm{X}$ & $\mathrm{X}$ & $\mathrm{X}$ & $\mathbf{R t}$ & \\
\hline
\end{tabular}

$\mathrm{X}$ : mineral identifed in sample.

(bt, grt, cri...): accessory present as inclusion in biotite, garnet, cordierite.

fels: felsic mineral (feldspar or quartz).

$\mathrm{m}$ : accessory present as matrix (or interstitial) mineral.

?: indicates tentative identification only.

Other mineral abbreviations after Kretz (1983).

and expected values was better than $10 \%$ for most of the REEs (Appendix A). Analyses of some metals (Cr, $\mathrm{Ni}, \mathrm{V})$ are strictly qualitative as they show larger inaccuracies. More details on LA-ICP-MS analytical techniques are in Otamendi et al. (2002).

\section{Texture of the accessory phases with increasing metamorphic grade}

Detailed SEM and microprobe analyses revealed that REE-Y-Th-U-Zr-rich accessories in granulites

Fig. 1. BSE images showing perographic features of accessory minerals. Scale bar in A to E is $10 \mu \mathrm{m}$. Scale bar in F is $20 \mu \mathrm{m}$, in $\mathrm{G}$ is $100 \mu \mathrm{m}$, and in $\mathrm{H}$ is $50 \mu \mathrm{m}$. (A) Complex and irregular zoning in type-III monazite with bright high Th (low U) core and high U (low Th) rim. This chemical zoning is the reverse of the common zoning in leucocratic migmatites. Sotosalbos granite 100941 (analyses 44 to 47 in Table 3 ). (B) Idiomorphic zoning in type-II monazites showing also an increase in U contents from core to rim. Sotosalbos granite 100560 (analyses 11 and 10 in Table 3). (C) Complex zoning in type-III monazite showing bright interstitial cheralite (Cher). In this grain, chemical zoning is from low $\mathrm{Th}-\mathrm{U}$ core towards higher Th-U rim. Sotosalbos granite 100563 (analysis 17 in Table 3). (D) Type-II xenotime in leucosome 101638 from Sotosalbos area. (E) Unzoned xenotime crystal (type-I) partially surrounding zircon. Layos granite 93193 . (F) Rounded sillimanite inclusion in unzoned zircon (type-D). Lower crustal granulitic xenolith 77750. (G) Type-A zircons with plagioclase inclusions in Sotosalbos granite 100943. (H) Type-B zircons in Layos granite (M-3 concentrate). 

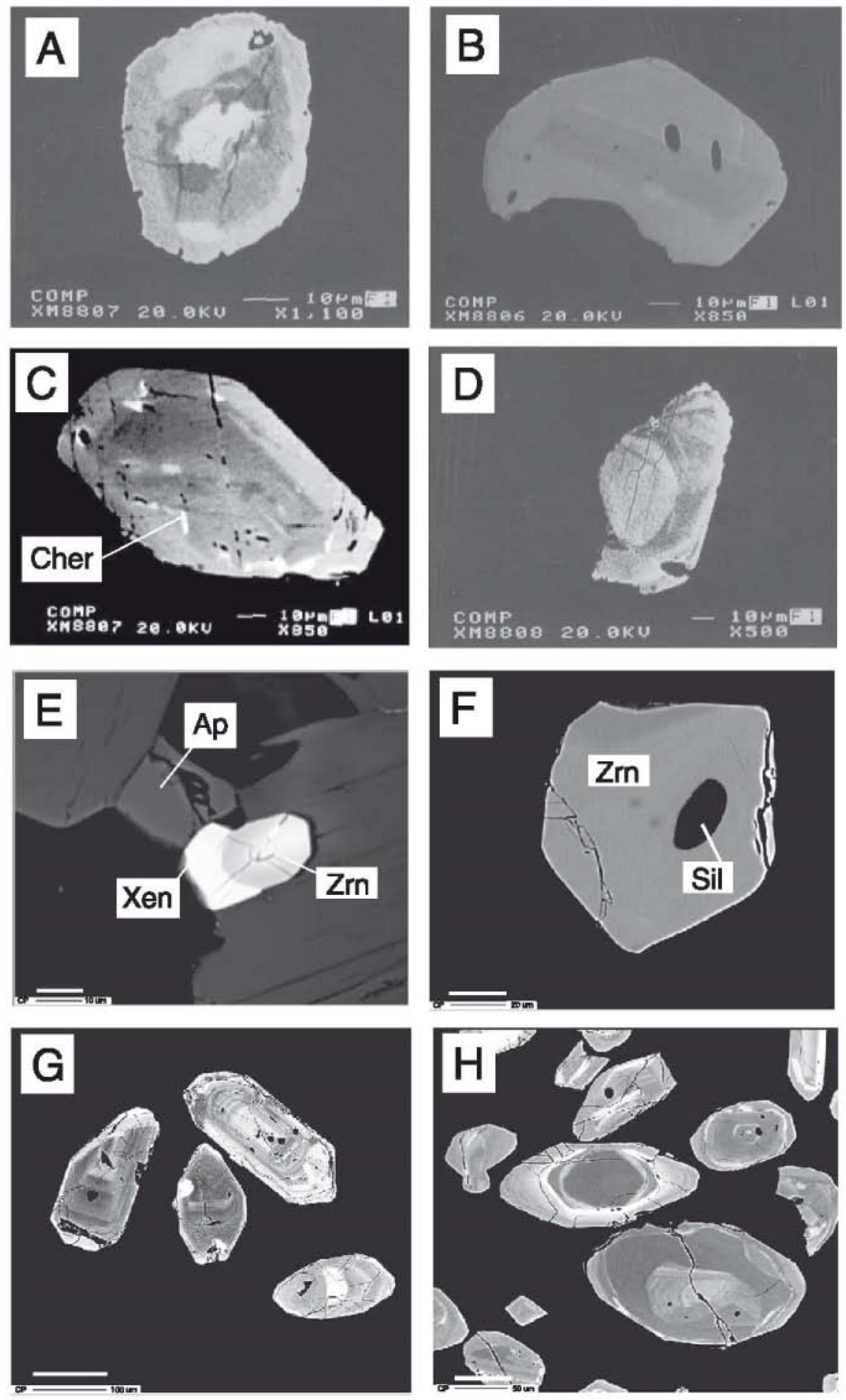


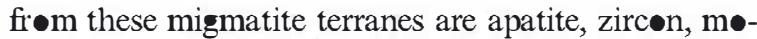
nazite and more rarely, xenotime (Table 1). In lower crustal xenoliths, only rare apatite in some enclaves, zircon and scarce monazite, are found. The general trend of a decrease in abundance of accessory minerals in peraluminous granulites with increasing metamorphic grade is similar to that found by Bea and Montere (1999) in the Ivrea-Verbane section or by Pyle and Spear (1999) with respect to xenotime in pelites. This contrasts with the general trend of increased growth of accessory minerals usually found in peraluminous lithøløgies frøm greenschist to amphibølite/granulite facies transition (Franz et al., 1996; Rubbatto et al., 2001). In general, accessory phases in the studied samples appear as isølate crystals not defining clusters or groups as found in felsic igneous rocks (e.g. Wark and Miller, 1993) or in accessøry-rich metamorphic rocks (e.g. Pan, 1997). They usually appear as

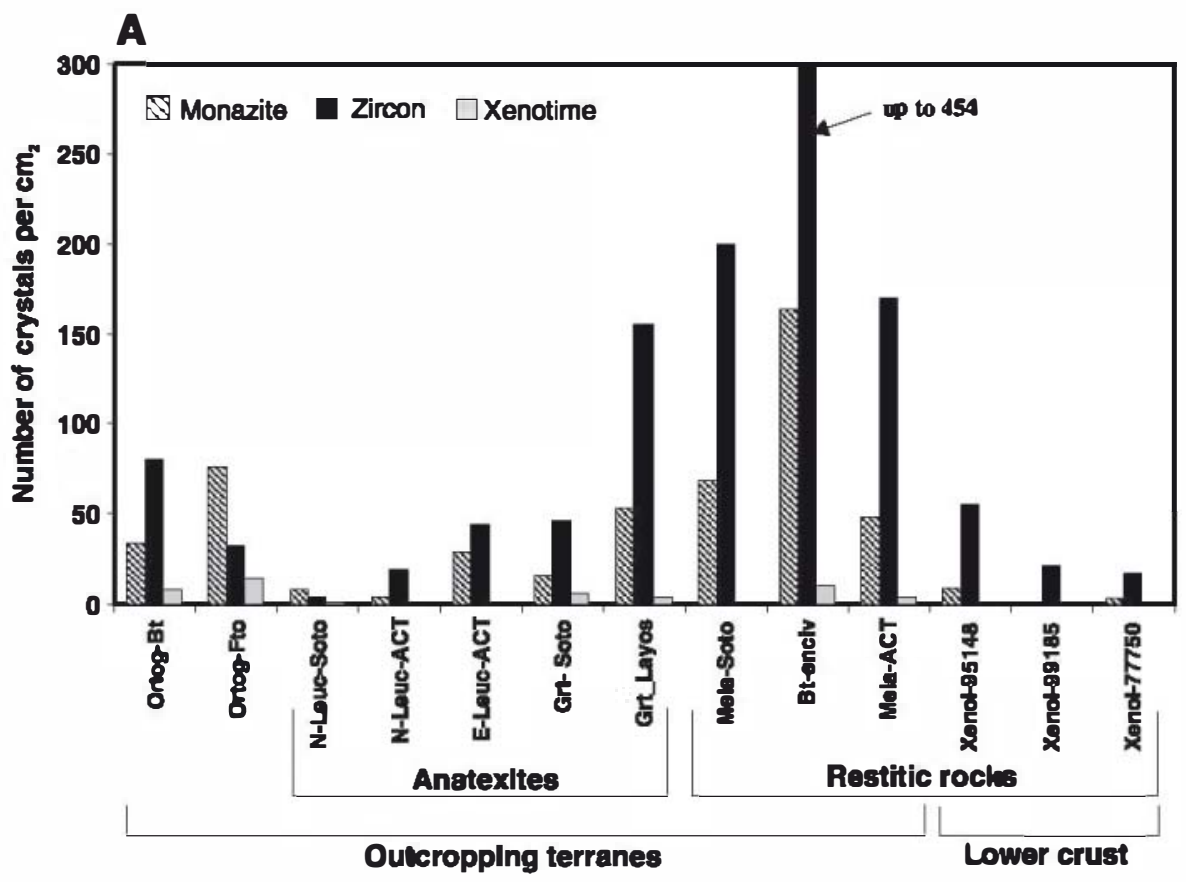

B

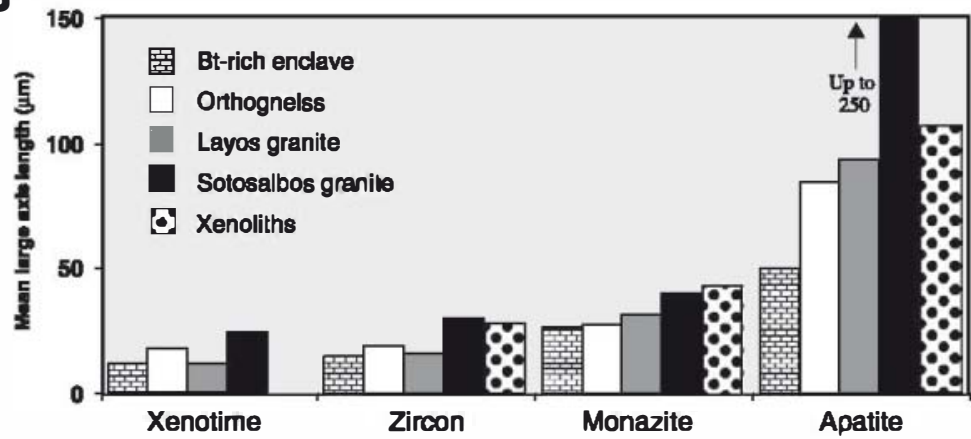

Fig. 2. Variations in modal amounts and average large axis length of accessory minerals in granulitic samples. (A) Modal amounts of monazite, zircon and xenotime in melt-rich migmatites (anatexites), residuum-rich migmatites and regional orthogneisses. Xenolith samples show a marked decrease in abundance of accessory minerals with respect to restitic migmatites from granulite terranes. Xenotime is absent in xenoliths and monazite is very scarce in some thin sections. Data set from Table 1. (B) Average large axis length of accessory phases in granulitic samples showing an increase in size from the smallest (xenotime) towards the largest (apatite) crystals irrespectively of the lithotype. 

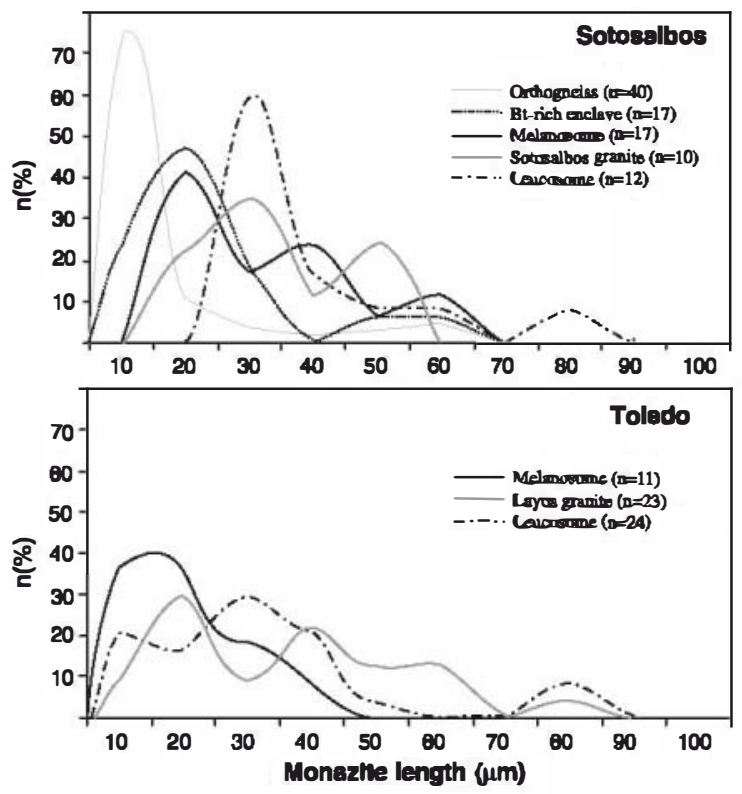

Fig. 3. Plots showing monazite crystal size distribution in samples from the studied migmatite terranes. A larger crystal size and a decrease overall monazite frequency is characteristic of leucocratic migmatites, best displayed in monazites from the Sotosalbos complex. The legend shows the number of measured monazite crystals.

interstitial $\bullet$ matrix minerals but are alsø inclusiøns in the majør phases (Table 2). Nevertheless, søme crystals of accessøry minerals •ccasionally appear grøuped, mostly in søme accessøry-rich samples (Fig. 1E).

\subsection{Apatite}

Apatite is the largest accessory mineral ( $>95 \mu \mathrm{m}$ in melt-rich migmatites) (Fig. 2). Its prop॰rtion diminishes strongly from samples of granulite terranes to those of granulitic xenoliths (Table 1). There is alsø a modal decrease from residuum-rich to melt-rich migmatites. Monazite may surround apatite crystals $\bullet$ be included in larger apatites. More rarely, xenotime and zircon are situated at apatite rims (Fig. 1E). Due to their larger size, although there are few apatite crystals in a $1-\mathrm{cm}^{2}$ section (Table 1 ), they usually constitute the most abundant accessory mineral.

\subsection{Monazite}

The modal amount of monazite diminishes significantly from migmatites of granulite terranes to gran- ulitic xenøliths (Table 1, Fig. 2). There is alsø a marked decrease of monazite abundance between leucosøme and melanosøe pairs, as usually described in migmatite terranes (e.g. Watt et al., 1996; Nabeleck and Glascock, 1995; Bea and Monter,, 1999). A maximum concentration in biotite-rich enclaves of Sotosalbos granitoid is observed, in agreement with the
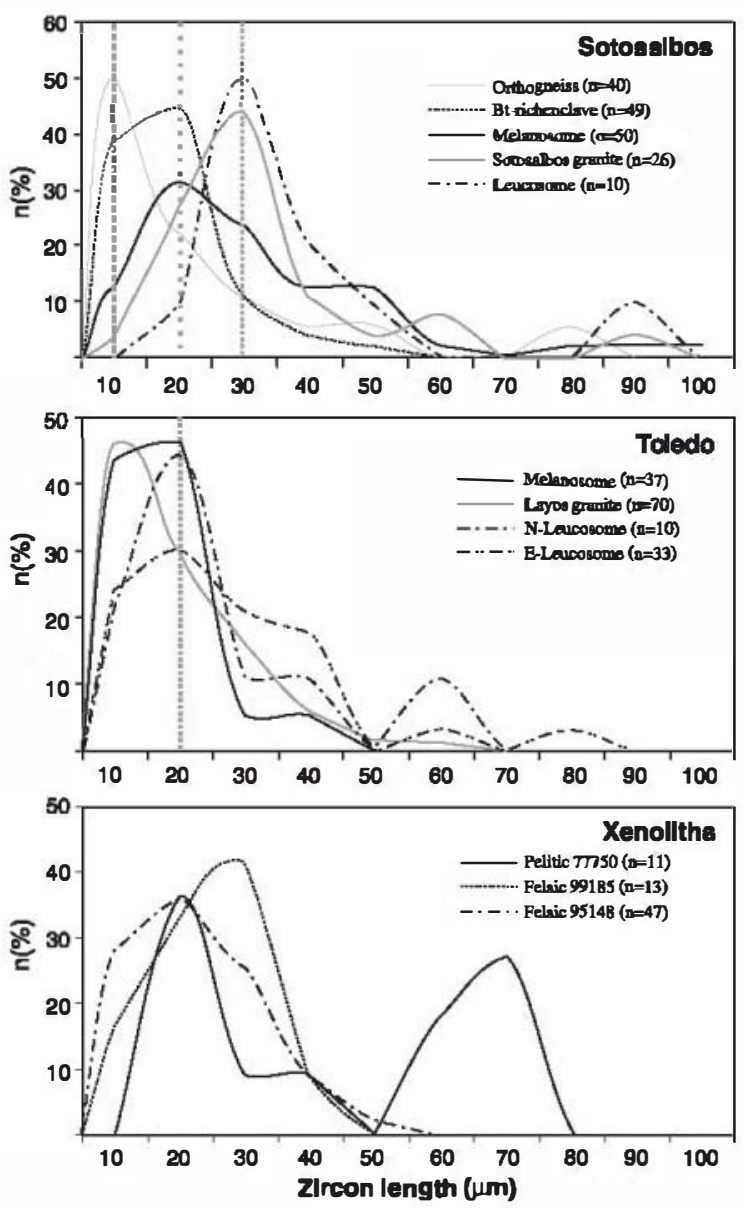

Fig. 4. Plots showing zircon crystal size distribution from studied samples. As for monazites, zircons have a larger crystal size and a higher heterogeneity of sizes in leucocratic migmatites. In the Sotosalbos plot, vertical line at $30 \mu \mathrm{m}$ approximates the modal zircon length in melt-rich migmatites (e.g. leucosomes), whereas 20 $\mu \mathrm{m}$ is the modal zircon length in melt-poor migmatites (bt-rich enclave and melanosome), and $10 \mu \mathrm{m}$ is the modal zircon length in regional orthogneisses. In the plot of Toledo zircons, vertical line at $20 \mu \mathrm{m}$ approximates the mode in melt-rich migmatites whereas restite-rich migmatites have modal zircon lengths more displaced towards lesser size (being $13 \mu \mathrm{m}$ in average). $n$ =number of measured zircon crystals. 
Table 3

Representative EMP analyses of monazites

\begin{tabular}{|c|c|c|c|c|c|c|c|c|c|c|c|c|c|c|c|c|c|c|c|}
\hline Sample & Anal & & & $\mathrm{La}_{2} \mathrm{O}_{3}$ & $\mathrm{Ce}_{2} \mathrm{O}_{3}$ & $\mathrm{Pr}_{2} \mathrm{O}_{3}$ & $\mathrm{Nd}_{2} \mathrm{O}_{3}$ & $\mathrm{Sm}_{2} \mathrm{O}_{3}$ & $\mathrm{Gd}_{2} \mathrm{O}_{3}$ & $\mathrm{Dy}_{2} \mathrm{O}_{3}$ & $\mathrm{Er}_{2} \mathrm{O}_{3}$ & $\mathrm{Yb}_{2} \mathrm{O}_{3}$ & $\mathrm{Y}_{2} \mathrm{O}_{3}$ & $\mathrm{ThO}_{2}$ & $\mathrm{UO}_{2}$ & $\mathrm{P}_{2} \mathrm{O}_{5}$ & $\mathrm{SiO}_{2}$ & $\mathrm{CaO}$ & Total \\
\hline \multicolumn{20}{|c|}{ Sotosalbos Complex } \\
\hline 100560 & Granite & 10 & $\mathrm{r}$ & 13.12 & 27.4 & 2.73 & 11.85 & 1.92 & 2.36 & 0.59 & 0.30 & $\bullet .11$ & 2.34 & 4.48 & 2.15 & 30.78 & 0.06 & 1.34 & 101.79 \\
\hline 100560 & Granite & 11 & $\mathrm{c}$ & 13.76 & 27.92 & 2.88 & 12.58 & 1.78 & 2.33 & $\bullet .53$ & 0.28 & $\bullet .12$ & 2.46 & 4.11 & 0.29 & 30.15 & 0.08 & 0.92 & 100.29 \\
\hline 100941 & Granite & 44 & bc & 13.60 & 25.37 & 2.70 & 9.20 & 1.27 & 1.86 & 0.47 & 0.29 & $\bullet .13$ & 1.2 & 11.73 & 0.94 & 27.34 & 1.70 & 1.31 & 99.53 \\
\hline 100941 & Granite & 47 & $r$ & $13 . \bullet 3$ & 26.91 & 3.15 & 11.59 & 2.11 & 2.15 & $\bullet .62$ & 0.19 & $\bullet .12$ & 2.16 & 3.72 & 4.34 & $3 \bullet .19$ & 0.05 & 1.56 & 102.32 \\
\hline 100563 & Granite & 17 & Cheralite & 8.74 & 17.23 & 2.07 & 8.44 & 1.25 & 1.42 & $\bullet .3 \bullet$ & 0.32 & $\bullet .15$ & 1.43 & 29.54 & 2.49 & 21.96 & 5.96 & 1.44 & 103.07 \\
\hline 100563 & Granite & 20 & $\mathrm{r}$ & 13.39 & 25.92 & 2.97 & 11.50 & 2.09 & 2.33 & 0.91 & 0.39 & $\bullet .15$ & 2.92 & 4.43 & 2.4 & 30.18 & $\bullet .11$ & 1.35 & 101.37 \\
\hline 100940 & Granite & 22 & $\mathrm{c}$ & 11.91 & 26.00 & 3.10 & 12.32 & 1.97 & 2.48 & 0.79 & $\bullet .37$ & 0.09 & 1.94 & 5.47 & 0.62 & 30.78 & 0.19 & 1.13 & 99.67 \\
\hline 100940 & Granite & 23 & $\mathrm{r}$ & 11.63 & 25.90 & 3.06 & 12.31 & 1.97 & 2.17 & 0.86 & 0.34 & $\bullet .15$ & 1.95 & 5.53 & 0.68 & 31.04 & 0.20 & 1.13 & 99.50 \\
\hline 60870 & Orthogneiss & 15 & unz & 11.64 & 26.04 & 2.97 & 11.87 & 2.00 & 2.34 & 1.02 & 0.47 & $\bullet .17$ & 4.12 & 6.43 & $\bullet .7 \bullet$ & 30.81 & 0.20 & 1.35 & 102.71 \\
\hline 60870 & Orthogneiss & 1 & $\mathrm{c}$ & 9.11 & 24.55 & 3.00 & 10.98 & 2.4 & 2.24 & 0.96 & 0.43 & 0.2 & 3.43 & 9.53 & 1.91 & 30.20 & 0.16 & 2.44 & 102.10 \\
\hline 100942 & Orthogneiss & 44 & unz & 11.87 & 25.58 & 2.80 & 11.45 & 1.99 & 2.17 & $\bullet .61$ & 0.29 & $\bullet .13$ & 2.20 & 6.46 & 1.57 & 28.36 & 0.2 & 1.69 & 97.69 \\
\hline 100942 & Orthogneiss & 53 & unz & 13.05 & 26.46 & 2.73 & 11.56 & 1.94 & 2.36 & 0.72 & $\bullet .31$ & $\bullet .13$ & 2.36 & 5.26 & 2.34 & 30.96 & $\bullet .17$ & 1.47 & 102.06 \\
\hline 62458 & Orthogneiss & 20 & unz & 12.58 & 27.46 & 3.11 & 12.54 & 2.04 & 2.26 & 0.82 & $\bullet .36$ & 0.14 & 3.38 & 4.87 & 0.46 & $31.3 \bullet$ & 0.18 & 1.08 & 103.05 \\
\hline 62458 & Orthogneiss & 23 & unz & 12.97 & 27.67 & 3.09 & 12.05 & 2.05 & 2.31 & & 0.25 & 0.07 & 2.11 & 4.96 & 2.01 & 31.34 & 0.04 & 1.52 & 103.39 \\
\hline 100945 & Orthogneiss & 19 & $\mathrm{c}$ & 10.90 & 23.81 & 2.82 & 11.50 & 1.99 & 2.29 & 1.03 & 0.44 & $\bullet .13$ & 4.31 & 7.38 & 1.98 & 28.92 & 0.73 & 1.24 & 100.12 \\
\hline 101638 & Leucosome & 25 & $\mathrm{i}$ & 13.29 & 25.82 & 2.77 & 10.92 & 1.75 & 2.14 & 0.82 & 0.44 & 0.10 & 2.93 & 4.97 & 3.18 & 28.98 & $\bullet .11$ & 1.60 & 100.38 \\
\hline 101638 & Leucosome & 26 & bi & 13.23 & 26.66 & 2.75 & 10.94 & 1.73 & 2.19 & 0.43 & 0.38 & 0.08 & 2.33 & 7.4 & 1.92 & 28.78 & 0.95 & 1.13 & 101.34 \\
\hline 101638 & Leucosome & 34 & $\mathrm{bc}$ ? & 12.77 & 24.99 & 2.59 & 10.47 & 1.59 & 2.22 & 0.77 & 0.45 & $\bullet .12$ & 3.18 & 5.79 & 3.69 & 30.00 & $\bullet .12$ & 1.88 & 101.22 \\
\hline 101638 & Leucosome & 45 & unz & 13.74 & 27.11 & 2.79 & 10.75 & 1.37 & 2.22 & $\bullet .53$ & $\bullet .36$ & $\bullet .11$ & 2.06 & 6.74 & 1.22 & 28.56 & 0.95 & 1.12 & 99.85 \\
\hline 102183 & Leucogranite & 31 & $\mathrm{c}$ & 12.66 & 25.22 & 2.92 & 11.13 & 1.95 & 2.35 & 0.69 & 0.49 & $\bullet .15$ & 1.82 & 5.20 & 3.45 & 30.78 & 0.10 & 1.72 & 101.23 \\
\hline 102183 & Leucogranite & 32 & $\mathrm{i}$ & 13.13 & 26.21 & 2.89 & 11.39 & 1.91 & 2.47 & $\bullet 6$ & 0.38 & 0.10 & 1.71 & 4.68 & 2.58 & 29.24 & $\bullet .11$ & 1.46 & 99.46 \\
\hline 102183 & Leucogranite & 30 & c & 12.44 & 25.22 & 2.86 & 10.93 & 2.04 & 2.16 & $\bullet .63$ & 0.53 & $\bullet .12$ & 1.63 & 4.71 & 4.74 & 29.79 & $\bullet .13$ & 1.86 & 100.39 \\
\hline 102183 & Leucogranite & 29 & $r$ & 12.33 & 24.97 & 2.83 & 11.17 & 2.06 & 2.45 & $\bullet .67$ & 0.54 & $\bullet .16$ & 1.87 & 4.41 & 3.94 & 29.11 & 0.07 & 1.65 & 98.87 \\
\hline 102181 & Leucosome & 8 & $\mathrm{c}$ & 10.09 & 24.23 & 2.95 & 11.03 & 2.34 & 2.32 & 0.77 & 0.47 & $\bullet .10$ & 1.56 & 8.56 & 2.78 & 29.42 & 0.21 & 2.34 & 99.82 \\
\hline 102181 & Leucosome & 9 & $r$ & 12.65 & 25.64 & 3.04 & 12.30 & 1.98 & 2.25 & $\bullet .61$ & 0.52 & 0.10 & 1.37 & 5.37 & 1.64 & 29.51 & 0.10 & 1.54 & 99.21 \\
\hline 102178 & Leucosome & 16 & unz & 13.52 & 26.62 & 3.18 & 13.55 & 1.85 & 1.88 & - .41 & 0.47 & 0.10 & 1.53 & 4.80 & 0.25 & 29.71 & $\bullet .15$ & 1.00 & 99.36 \\
\hline 102178 & Leucosome & 15 & unz & 13.59 & 26.15 & 2.87 & 11.51 & 1.94 & 2.23 & $\bullet .62$ & 0.52 & 0.10 & 1.72 & 4.93 & 1.55 & 29.85 & $\bullet .11$ & 1.43 & 99.58 \\
\hline 102175 & Melanosome & 29 & $\mathrm{c}$ & 13.39 & 27.31 & 3.02 & 12.63 & 1.73 & 2.03 & 0.64 & 0.49 & $\bullet .13$ & 2.95 & 4.62 & 0.4 & 28.92 & 0.16 & 1.08 & 99.92 \\
\hline 102175 & Melanosome & 30 & $r$ & 12.4 & 26.33 & 2.90 & 12.54 & 1.79 & 2.23 & 0.78 & 0.49 & $\bullet .12$ & 3.66 & 5.47 & 0.55 & 28.96 & 0.24 & 1.24 & 100.11 \\
\hline 102175 & Melanosome & 31 & $\mathrm{c}$ & 13.42 & 27.08 & 2.92 & 13.14 & 1.80 & 2.13 & 0.58 & 0.49 & $\bullet .11$ & 2.73 & 4.27 & 0.54 & 29.17 & 0.10 & 1.06 & 100.00 \\
\hline 102175 & Melanosome & 32 & $r$ & 13.16 & 26.97 & 3.03 & 13.00 & 1.76 & 2.17 & 0.58 & 0.46 & $\bullet .11$ & 3.12 & 4.78 & 0.4 & 29.03 & 0.17 & 1.02 & 100.26 \\
\hline 100562 & Bt-rich enclave & 10 & $\mathrm{c}$ & 13.28 & 27.33 & 3.06 & 13.05 & 1.71 & 2.00 & 0.46 & 0.51 & 0.08 & 1.73 & 4.49 & 0.67 & 29.11 & 0.09 & 1.25 & 99.23 \\
\hline 100562 & Bt-rich enclave & 11 & $\mathrm{r}$ & 13.06 & 26.52 & 3.06 & 12.76 & 1.92 & 2.15 & $0.7 \bullet$ & 0.53 & 0.09 & 2.73 & 3.11 & 1.43 & 29.36 & 0.07 & 1.08 & 99.12 \\
\hline 100562 & Bt-rich enclave & 18 & unz & 13.54 & 27.55 & 3.04 & 12.79 & 1.84 & 2.14 & 0.64 & 0.46 & $\bullet .15$ & 3.01 & 3.4 & 1.30 & 30.42 & 0.05 & 1.01 & 101.90 \\
\hline
\end{tabular}




\begin{tabular}{|c|c|c|c|c|c|c|c|c|c|c|c|c|c|c|c|c|c|c|c|}
\hline \multicolumn{20}{|c|}{ Toledo Complex } \\
\hline 87085 & Kinzigite & 2 & in-git & 13.24 & 26.04 & 2.53 & 10.02 & 3.51 & 3.13 & 0.88 & 0.00 & $\bullet .61$ & 3.17 & 4.47 & $\bullet .53$ & 30.02 & $\bullet .15$ & 1.08 & 99.81 \\
\hline 87085 & Kinzigite & 4 & in-cra & 14.39 & 28.63 & 2.87 & 10.90 & 3.56 & 2.90 & 0.00 & 0.00 & 0.02 & $\bullet .16$ & 4.82 & 0.58 & 29.77 & 0.42 & 0.87 & 100.29 \\
\hline 87085 & Kinzigite & 12 & $\mathrm{c}$ & 14.19 & 27.50 & 2.21 & 9.86 & 3.59 & 3.26 & 0.69 & 0.39 & 0.4 & 2.55 & 2.45 & $\bullet .51$ & 30.11 & $\bullet .13$ & 0.68 & 98.99 \\
\hline 87085 & Kinzigite & 13 & $r$ & 14.78 & 28.81 & 2.41 & 10.52 & $3.7 \bullet$ & 2.95 & 0.18 & 0.65 & 0.30 & 0.62 & 2.46 & 0.43 & 29.61 & $\bullet .11$ & 0.58 & 98.46 \\
\hline 93198 & Melanosome & 1 & & 13.75 & 30.24 & nd & 10.31 & 1.80 & $1.6 \bullet$ & $\bullet .67$ & 0.50 & 0.14 & 2.17 & 4.30 & 0.97 & 31.78 & 0.10 & 1.13 & 100.06 \\
\hline 93198 & Melanosome & 7 & & 14.98 & 31.59 & $\mathrm{nd}$ & 10.45 & 2.03 & 1.68 & 0.46 & 0.35 & 0.14 & 1.36 & 4.03 & 0.80 & 31.63 & 0.08 & 0.98 & 100.68 \\
\hline 93198 & Melanosome & 9 & & 13.56 & 29.63 & nd & 9.87 & 2.02 & 2.21 & 1.04 & 0.34 & 0.10 & 2.59 & 3.84 & 0.68 & 30.28 & 0.15 & 1.03 & 97.79 \\
\hline 93198 & Melanosome & 10 & & 13.46 & 29.42 & nd & 9.87 & 1.95 & 1.65 & 0.95 & 0.37 & 0.10 & 2.15 & 4.20 & 1.02 & 29.98 & 0.17 & 1.10 & 96.65 \\
\hline 81926 & Leucogranite & 1 & $\mathrm{c}$ & 12.95 & 29.57 & $\mathrm{nd}$ & 11.93 & 2.26 & 2.24 & $\bullet .72$ & 0.25 & 0.09 & 1.34 & 3.56 & 1.96 & 30.26 & 0.04 & 1.08 & 98.74 \\
\hline 81926 & Leucogranite & 3 & $\mathrm{r}$ & 15.18 & 32.41 & nd & 11.63 & 1.62 & 0.66 & 0.20 & 0.29 & 0.08 & 0.41 & 5.87 & 0.98 & 29.79 & 0.08 & 1.43 & 100.89 \\
\hline 81926 & Leucogranite & 16 & $\mathrm{c}$ & 15.52 & 32.71 & nd & 11.31 & 1.51 & 0.77 & 0.15 & 0.29 & 0.10 & 0.43 & 6.00 & 0.98 & 30.13 & 0.09 & 1.47 & 101.72 \\
\hline 81926 & Leucogranite & 17 & $\mathrm{r}$ & 14.98 & 31.05 & nd & 10.49 & 1.10 & 1.05 & 0.42 & 0.35 & 0.12 & 1.34 & 5.64 & 1.22 & 29.84 & 0.08 & 1.44 & 99.47 \\
\hline 87202 & Leucogranite & 49 & $\mathrm{c}$ & 13.45 & 27.62 & 2.21 & 10.44 & 1.6 & 2.09 & $\bullet .35$ & 0.18 & 0.04 & 1.55 & 6.52 & 1.49 & 30.22 & 0.18 & 1.75 & 100.00 \\
\hline 87202 & Leucogranite & 51 & $\mathrm{r}$ & 13.47 & 28.22 & 2.12 & 10.82 & 1.74 & 2.02 & 0.18 & $\bullet .03$ & 0.02 & 0.68 & 7.51 & 1.31 & 30.13 & 0.32 & 1.80 & 100.40 \\
\hline 87202 & Leucogranite & 52 & $\mathrm{c}$ & 12.41 & 27.65 & 2.18 & 11.68 & 1.64 & 1.67 & 0.32 & $\bullet .16$ & 0.07 & $\bullet .54$ & 9.00 & $\bullet .67$ & 29.52 & 0.64 & 1.69 & 100.03 \\
\hline 87202 & Leucogranite & 53 & $\mathrm{r}$ & 15.03 & 28.89 & 1.82 & 9.10 & 1.19 & 1.90 & 0.38 & 0.00 & 0.00 & 0.92 & 6.13 & 3.06 & 30.08 & $\bullet .13$ & 1.91 & 100.69 \\
\hline 93197 & E-leucosome & 32 & $\mathrm{c}$ & 11.15 & 26.36 & 2.43 & 11.38 & 1.91 & 1.92 & 0.17 & 0.19 & 0.07 & 0.71 & 9.93 & 1.14 & 29.26 & 0.36 & 2.26 & 99.42 \\
\hline 93197 & E-leucosome & 33 & $\mathrm{r}$ & 13.21 & 28.43 & 2.19 & 11.15 & 2.06 & 2.52 & 0.42 & 0.09 & $\bullet .13$ & 0.81 & 5.80 & 1.08 & 29.21 & 0.30 & 1.51 & 98.99 \\
\hline 93197 & E-leucosome & 46 & $\mathrm{c}$ & 13.91 & 28.67 & 2.11 & 11.04 & 1.88 & 2.44 & $\bullet .39$ & $\bullet .31$ & $\bullet .12$ & 1.43 & 5.01 & 0.77 & 29.45 & 0.15 & 1.22 & 99.04 \\
\hline 93197 & E-leucosome & 47 & $\mathrm{i}$ & 10.91 & 25.64 & 2.30 & 10.91 & 2.08 & 1.96 & 0.48 & 0.16 & $\bullet .11$ & 1.20 & 8.61 & 2.94 & 29.59 & 0.23 & 2.38 & 99.75 \\
\hline 93197 & E-leucosome & 48 & $r$ & 13.24 & 27.36 & 1.97 & 10.55 & 1.79 & 2.14 & $\bullet .51$ & $\bullet .15$ & 0.05 & 1.99 & 5.74 & 1.22 & 29.52 & 0.10 & 1.60 & 98.09 \\
\hline 93193 & Layos granite & 5 & $\mathrm{c}$ & 13.62 & 29.27 & 2.2 & 11.92 & 2.15 & 2.62 & $\bullet .61$ & 0.22 & $\bullet .13$ & 1.61 & 3.45 & 0.96 & 29.91 & 0.06 & 0.94 & 99.91 \\
\hline 93193 & Layos granite & 6 & $\mathrm{r}$ & 13.27 & 28.18 & 2.08 & 11.19 & 1.59 & 2.23 & $\bullet .35$ & 0.06 & 0.03 & $\bullet .7 \bullet$ & 6.48 & 2.03 & 30.63 & 0.07 & 1.85 & 100.79 \\
\hline 93193 & Layos granite & 16 & $\mathrm{c}$ & 13.29 & 28.94 & 2.24 & 11.34 & 1.38 & 1.65 & 0.12 & $\bullet .11$ & 0.00 & 0.43 & 8.56 & - .41 & 28.50 & 1.00 & 1.24 & 99.24 \\
\hline 93193 & Layos granite & 17 & $r$ & 11.13 & 24.26 & 1.76 & 9.74 & 1.4 & 2.13 & 0.56 & $\bullet .17$ & $\bullet .11$ & 2.14 & 4.73 & 1.51 & 27.26 & 5.72 & 1.42 & 94.25 \\
\hline \multicolumn{20}{|c|}{ Xen•liths } \\
\hline 77750 & Pelitic & 5 & $\mathrm{c}$ & 13.44 & 29.85 & 2.80 & 11.33 & 2.78 & 2.10 & 0.00 & 0.00 & 0.29 & 0.04 & 5.02 & $\bullet .7 \bullet$ & 29.27 & 0.65 & 0.87 & 99.52 \\
\hline 77750 & Pelitic & 6 & $\mathrm{r}$ & 13.67 & 29.37 & 2.71 & 10.73 & 2.4 & 2.05 & 0.04 & 0.00 & 0.18 & 0.08 & 6.02 & 0.39 & 28.56 & 0.62 & 0.89 & 98.26 \\
\hline 77750 & Pelitic & 8 & unz & 13.34 & 29.43 & 2.67 & 11.22 & 2.79 & 1.99 & 0.00 & 0.00 & 0.04 & $\bullet .03$ & 4.72 & 0.89 & 29.56 & 0.45 & 0.89 & 98.31 \\
\hline $\mathrm{U}-46$ & Pelitic & 1 & unz & 14.80 & 34.09 & 0.73 & 12.20 & 1.4 & $\bullet .61$ & 0.00 & 0.04 & $\bullet .15$ & $\bullet .01$ & 5.26 & $\bullet .31$ & 30.06 & - .21 & 1.13 & 101.00 \\
\hline$U-46$ & Pelitic & 2 & unz & 15.52 & 33.64 & 0.00 & 10.63 & 1.64 & 0.67 & 0.01 & 0.30 & $\bullet .12$ & 0.04 & 5.29 & 0.37 & 30.54 & 0.19 & 1.06 & 100.13 \\
\hline 81846 & Felsic & 24 & unz & 15.31 & 31.85 & 3.78 & 13.70 & 2.32 & 1.93 & 0.15 & 0.05 & 0.08 & 0.24 & 1.01 & 0.24 & 30.80 & $\bullet .06$ & $\bullet .31$ & 101.90 \\
\hline 81846 & Felsic & 29 & unz & 10.74 & 27.25 & 3.70 & 16.45 & 2.58 & 0.95 & 0.00 & $\bullet .11$ & 0.07 & $\bullet .16$ & 6.51 & 0.73 & 29.61 & $\bullet .15$ & 1.44 & 100.55 \\
\hline 81846 & Felsic & 30 & unz & 9.15 & 23.17 & 3.30 & 14.41 & 2.15 & 0.40 & 0.09 & 0.08 & $\bullet .13$ & 0.09 & 13.70 & 0.94 & 29.61 & 0.48 & 2.79 & 100.55 \\
\hline 95148 & Felsic & 10 & $r$ & 8.52 & 23.83 & 3.68 & 16.22 & 1.34 & 0.08 & 0.09 & 0.10 & 0.05 & 0.08 & 15.24 & 1.20 & 26.08 & 2.46 & 1.27 & 100.29 \\
\hline 85148 & Felsic & 9 & $\mathrm{c}$ & 8.54 & 23.31 & 3.70 & 16.31 & 1.56 & 0.00 & 0.02 & 0.08 & 0.05 & 0.02 & 15.59 & 1.29 & 25.80 & 2.23 & 1.26 & 99.82 \\
\hline 99185 & Felsic & 1 & unz & 15.33 & 28.87 & 3.31 & 12.12 & 1.81 & 2.21 & 0.04 & 0.06 & $\bullet .11$ & 0.23 & 4.83 & 1.20 & 30.31 & 0.20 & 0.83 & 101.42 \\
\hline 95151 & Felsic & 16 & $\mathrm{c}$ & 14.13 & 27.80 & 3.38 & 13.68 & 1.99 & 1.52 & 0.26 & 0.06 & 0.03 & $\bullet .36$ & 3.99 & 0.97 & 29.19 & $\bullet .15$ & 0.90 & 98.38 \\
\hline 95151 & Felsic & 17 & $r$ & 10. 41 & 24.62 & 3.49 & 15.37 & 1.80 & 0.52 & 0.06 & 0.07 & 0.07 & 0.09 & 10.25 & 0.81 & 29.73 & 0.50 & 1.93 & 99.73 \\
\hline
\end{tabular}

$\mathrm{r}=$ rim, $\mathrm{c}=$ core, $\mathrm{i}=$ intermediate zone, unz = unzoned crystal, $\mathrm{b}=$ bright, in-mineral = included mineral. 
general observation of biotite being the main høst mineral for accessory phases in migmatite terranes (e.g. Bea et al., 1994; Johannes et al., 1995). Nevertheless, in the ACT terrane where biotite was nearly consumed during dehydration reactions (Barberø, 1995), the accessories are distributed elsewhere in the majør minerals of the migmatite, especially in those generated in the incongruent melting reactions i.e., K-feldspar, cordierite and gamet (Table 2). This has been observed in other migmatite areas (e.g. Watt et al., 1996). Monazite has few inclusions, and only biotite, apatite and zircon have been identified.

The average grain size of monazite is approximately 30-100 $\mu \mathrm{m}$ in diameter and remains similar in all the studied granulites, in contrast to other crustal sections where its grain-size decreases with metamorphic grade (Bea and Monter,, 1999) (Fig. 2B). In detail, søme slight contrasts in shape and size appear in monazites from the migmatite terrane samples. Mønazite from the anatectic granitøids of Sotosalbos are slightly more idiomorphic and larger than in the surrounding orthøgneisses or restitic counterparts. Leucosøme-hosted monazites are characterise by being longer and having a wider range of crystal sizes than those from orthøgneiss (prot॰liths) or from residuum-rich migmatites (i.e., melanısømes) (Fig. 3). This pattern is als observed in the size distribution of zircon (Fig. 4) and has been attributed to grain coarsening via Otswald ripening (Nemchin et al., 2001). Although the crystal size distribution estimate in this work is based on measured maximum length in thin sections, which underestimates the real greatest radius of the crystal, for comparison purposes it may be considered as a good estimate.

Monazites in melt-rich migmatites show more complex concentric or patchy zoning than those in their proteliths (ørthøgneisses) (Fig. 1A-C). Larger crystals with more euhedral shape and complex zoning appear in melt-rich migmatites (i.e., leucosømes) than in the residual fractions (Fig. 3). Growth of monazite is suggested during the migmatization event. Three textural types of monazites can be distinguished (Table 2): (i) type-I, unzoned equant grains of small size, (ii) type-II, equant grains with simple and regular (idiomorphic) zoning, and (iii) type-III, more irregular grains with a more diffuse and complex zøning, sometimes concentric or patchy. Occasionally, søme type-III monazites in restite-rich granitoids (Fig. 1A,C) show corrode cores with reaction rims, but truncation of zoning or cuspate boundaries have not
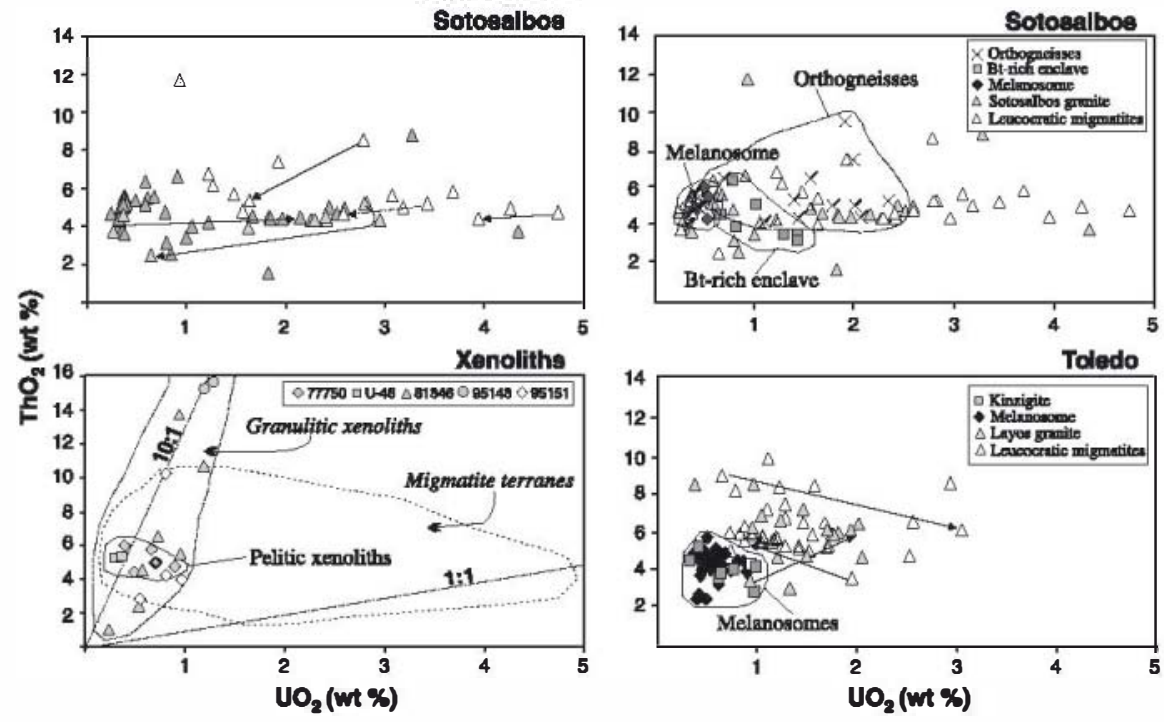

Fig. 5. U $\boldsymbol{B}_{2}$ and $\mathrm{Th} \boldsymbol{}_{2}$ variation in monatize. Compositional frelds of residuum-rich migmatites (melanosomes, bt-rich enclaves) are depicted. Chemical zoning is also marke by arrows from core to rim compositions (see data from Table 3). In the plot of xenoliths, the compositional feld of monazite from outcropping migmatite terranes is also shown for comparison. 
been $\bullet$ bserve in any monazite, as $\bullet$ ccurs in $\bullet$ ther granulite terranes (e.g. Zhu and O'Nions, 1999).

Type-I monazites are typical of orthøgneisses frøm Guadarrama, but als• appear in different granulitic samples together with type-III grains, which are the most common type (Table 2). These two monazite varieties are alsø found in granulite xenøliths. Type-II monazites with regular zoning have only been found in the Sotosalbos granitøid.

\subsection{Tenotime}

Yenotime is the least abundant of the studied accessory minerals. It does not appear in the granulitic xenoliths and is very scarce in the granulitic migmatites (Table 1). Hence, its modal abundance decreases rapidly with increasing metamorphic grade until total consumption, as $\bullet$ ccurs in $\bullet$ ther crustal sections with peraluminøus lithøløgies (Bea and Mønterø, 1999; Pyle and Spear, 1999). It appears as equant grains, variably corroded and locally asseciated with apatite or zircon grains (Fig. 1E). Xenøtime crystal size rarely exceeds $50 \mu \mathrm{m}$ in diameter (Fig. 2). Sieved textures have been $\bullet$ bserved, constituted by inclusions of major minerals. Two types of xenotime crystals are -bserved: (i) unzoned or (ii) with patchy or regular concentric zoning. This latter type usually appears in Sotosalbos leucosømes (Fig. 1D).

\subsection{Zircon}

Modal proportions of zircon decrease from granulitic terrane samples to the xenolith suite (Fig. 2). A slight increase in zircon length is •bserved between melt-rich and residuum-rich migmatites (Fig. 3), similar to the monazite size distribution. This zircon
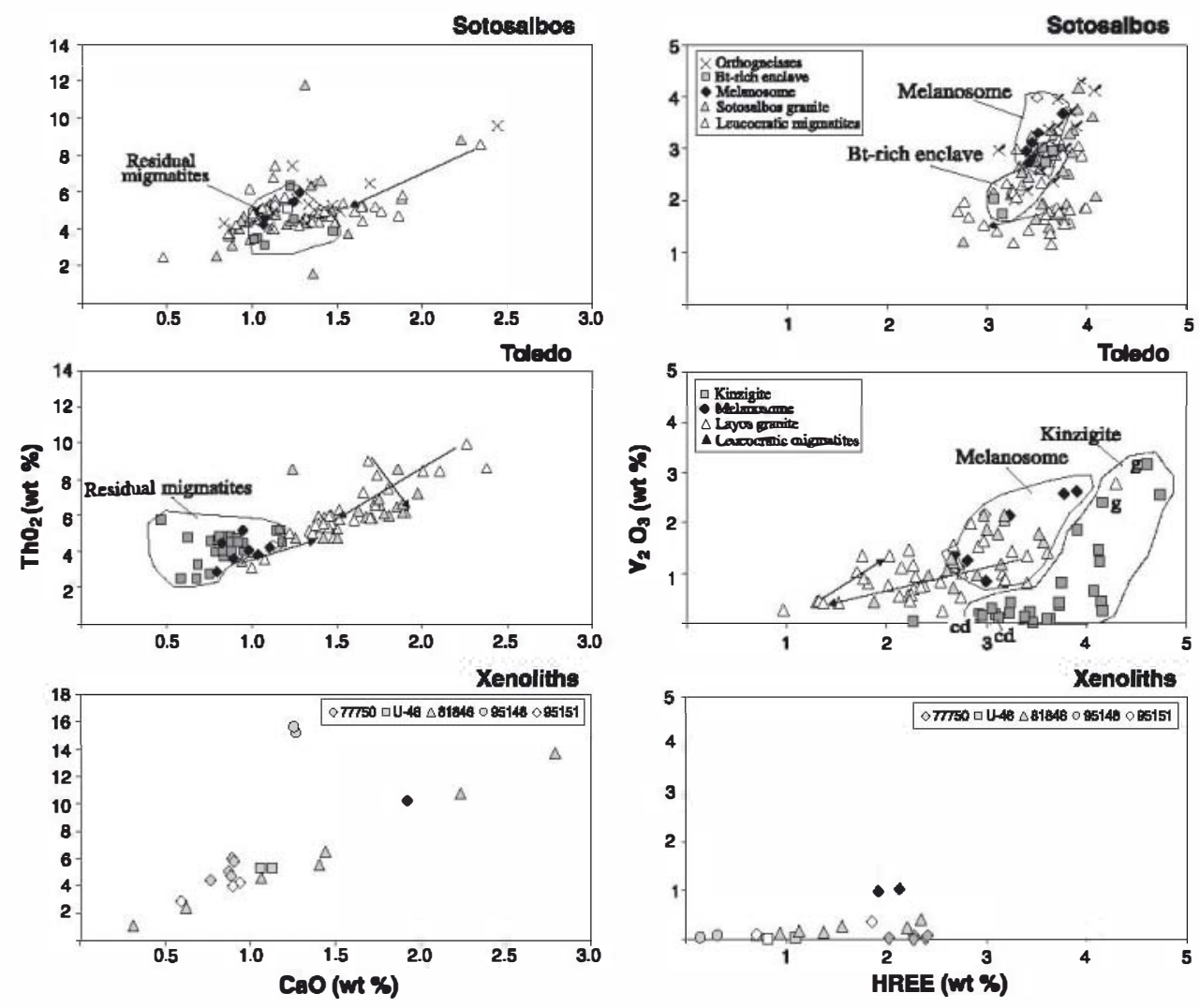

Fig. 6. Ca vs. Th $\boldsymbol{Q}_{2}$ and HREE vs. $\mathrm{Y}_{2} \boldsymbol{\bullet}_{3}$ variation in monazite. Monazites included in garnet (gt) or in their cordierite aureole (crd) from kinzigite sample are marked (see data from Table 3). Same symbols as in Fig. 5. 
Table 4

Representative EMP analyses of xenotimes

\begin{tabular}{|c|c|c|c|c|c|c|c|c|c|c|c|c|c|c|c|c|c|c|c|c|c|}
\hline Sample & Anal & & & $\mathrm{Ce}_{2} \mathrm{O}_{3}$ & $\mathrm{Pr}_{2} \mathrm{O}_{3}$ & $\mathrm{Nd}_{2} \mathrm{O}_{3}$ & $\mathrm{Sm}_{2} \mathrm{O}_{3}$ & $\mathrm{Gd}_{2} \mathrm{O}_{3}$ & $\mathrm{~Tb}_{2} \mathrm{O}_{3}$ & $\mathrm{Dy}_{2} \mathrm{O}_{3}$ & $\mathrm{Ho}_{2} \mathrm{O}_{3}$ & $\mathrm{Er}_{2} \mathrm{O}_{3}$ & $\mathrm{Yb}_{2} \mathrm{O}_{3}$ & $\mathrm{Lu}_{2} \mathrm{O}_{3}$ & $\mathrm{Y}_{2} \mathrm{O}_{3}$ & $\mathrm{ThO}_{2}$ & $\mathrm{UO}_{2}$ & $\mathrm{SiO}_{2}$ & $\mathrm{CaO}$ & $\mathrm{P}_{2} \mathrm{O}_{5}$ & Total \\
\hline \multicolumn{22}{|c|}{ Sotosalbus Complex } \\
\hline 6087 & Orthogneiss & 3 & 1 & 0.34 & $\bullet .06$ & 0.54 & 0.15 & 1.85 & 1.2 & 4.69 & 0.86 & 4.52 & 3.87 & 0.94 & 43.01 & 0.44 & 0.82 & 0.25 & 0.05 & 34.71 & 98.30 \\
\hline 60870 & Orthogneiss & 4 & $\mathrm{c}$ & 0.32 & $\bullet .06$ & 0.50 & 0.21 & 1.91 & 1.24 & 4.78 & 0.91 & 4.4 & 3.58 & 0.94 & 43.16 & 0.46 & $\bullet .62$ & 0.22 & 0.06 & 34.43 & 97.79 \\
\hline 6087 & Orthogneiss & 5 & $\mathrm{c}$ & $\bullet .35$ & 0.06 & 0.50 & 0.2 & 1.90 & 1.17 & 4.75 & 0.87 & 4.56 & 3.84 & 0.95 & 43.06 & $\bullet .21$ & 0.69 & $\bullet .14$ & $\bullet .10$ & 35.52 & 99.21 \\
\hline 100945 & Orthogneiss & 13 & $d z$ & 0.19 & $\bullet .06$ & 0.30 & 0.04 & 1.50 & 1.48 & 4.39 & 1.06 & 4.81 & 4.54 & 1.07 & 41.74 & $\bullet .15$ & 1.42 & 0.33 & 0.03 & 33.92 & 97.42 \\
\hline 100945 & Orthogneiss & 14 & $\mathrm{bz}$ & 0.25 & 0.06 & 0.30 & 0.12 & 1.62 & 1.48 & 4.38 & 1.02 & 4.52 & 4.35 & 1.00 & 41.02 & 0.47 & 3.57 & 0.72 & 0.07 & 34.30 & 99.69 \\
\hline 100560 & Granite & 3 & unz & 0.23 & 0.00 & 0.32 & 0.00 & 1.77 & 1.39 & 4.83 & 1.07 & 4.49 & 4.28 & 1.08 & 42.14 & 0.06 & 1.02 & $\bullet .16$ & 0.07 & 35.21 & 98.47 \\
\hline 10056 & Granite & 4 & unz & $\bullet .31$ & 0.03 & 0.52 & 0.18 & 1.86 & 1.52 & 4.91 & 1.16 & 4.54 & 4.15 & 1.01 & 42.43 & $\bullet .31$ & 0.69 & 0.23 & 0.07 & 34.68 & 98.98 \\
\hline 101638 & Leucosome & 27 & c & 0.26 & 0.05 & 0.28 & $\bullet .11$ & 1.69 & 2.92 & 4.26 & 0.76 & 3.43 & 3.47 & 1.49 & 42.39 & 0.20 & 2.83 & 0.41 & 0.37 & 33.44 & 98.75 \\
\hline 101638 & Leucosome & 28 & $r$ & 0.26 & 0.00 & 0.32 & $\bullet .12$ & 1.80 & 2.88 & 4.70 & 0.80 & 3.4 & 3.47 & 1.58 & 41.70 & 0.06 & 1.55 & 0.2 & 0.14 & 33.64 & 96.95 \\
\hline 102183 & Leucogranite & 28 & $d z$ & 0.22 & $\bullet .01$ & 0.26 & 0.15 & 1.94 & 2.95 & 4.03 & 0.66 & 3.68 & 3.09 & 1.69 & 43.04 & 0.04 & 1.54 & 0.27 & 0.14 & 33.99 & 98.12 \\
\hline 102183 & Leucogranite & 27 & $d z$ & 0.2 & 0.02 & 0.26 & $\bullet .11$ & 2.10 & 2.84 & 4.00 & $\bullet .67$ & 3.67 & 2.91 & 1.67 & 41.86 & 0.08 & 2.55 & 0.35 & $\bullet .17$ & 33.4 & 97.26 \\
\hline 102183 & Leucogranite & 26 & $\mathrm{bz} z$ & 0.27 & 0.04 & 0.35 & 0.14 & 1.88 & 2.72 & 3.84 & $\bullet .62$ & 3.48 & 2.86 & 1.54 & 40.34 & 0.39 & 6.43 & 0.99 & 0.27 & 32.07 & 98.80 \\
\hline 102183 & Leucogranite & 35 & $\mathrm{r}$ & 0.23 & 0.02 & 0.34 & 0.19 & 2.03 & 2.62 & 4.27 & $\bullet .66$ & 3.66 & 3.07 & 1.59 & 42.70 & 0.08 & 2.51 & 0.42 & $\bullet .16$ & 33.22 & 98.17 \\
\hline 102183 & Leucogranite & 33 & c & 0.36 & 0.09 & 0.59 & 0.28 & 2.00 & 2.62 & 4.03 & $\bullet .63$ & 3.51 & 3.00 & 1.46 & 40.88 & 0.50 & 4.29 & 0.89 & 0.24 & 32.90 & 98.75 \\
\hline 100562 & Bt-enclave & 1 & unz & 0.22 & 0.01 & 0.32 & 0.05 & 1.91 & 0.99 & 4.79 & 0.93 & 4.07 & 4.26 & 1.16 & 42.25 & 0.03 & 1.46 & 1.31 & 0.12 & 34.00 & 98.23 \\
\hline 100562 & Bt-enclave & 2 & unz & 0.22 & 0.00 & 0.38 & 0.06 & 2.05 & 1.09 & 4.99 & 0.96 & 4.21 & 4.72 & 1.27 & 42.30 & $\bullet .00$ & 1.38 & $\bullet .15$ & $\bullet .13$ & 35.79 & 100.12 \\
\hline \multicolumn{22}{|c|}{ Toledo Complex } \\
\hline 93193 & Layos granite & 1 & unz & $\bullet .30$ & 0.08 & 0.50 & $\bullet .19$ & $1.7 \bullet$ & 1.05 & 4.33 & 0.95 & 4.53 & 4.81 & 1.07 & 42.90 & $\bullet .11$ & 1.16 & 0.17 & 0.22 & 35.94 & 100.47 \\
\hline 93193 & Layos granite & 16 & unz & 0.30 & 0.00 & 0.50 & 0.20 & 1.83 & 1.13 & 4.71 & 1.07 & 4.33 & 4.62 & 1.11 & 41.36 & 0.03 & 0.86 & 0.29 & 0.24 & 32.98 & 96.02 \\
\hline
\end{tabular}

$\mathrm{r}=$ rim, $\mathrm{c}=$ core, $\mathrm{i}=$ intermediate zone, unz=unzoned crystal, $\mathrm{d} z=$ dark zone, $\mathrm{b} z=$ bright zone. 
coarsening in melt-rich migmatites has been als• -bserved in other migmatite terranes (Tanner and Behrmann, 1997; Nemchin et al., 2001).

The scarcity of igneous A-type zircons (after Watt et al., 1996), with complex and concentrically regular zoning, in the studied granulitic samples is remarkable. Nevertheless, A-type zircons $(>100 \mu \mathrm{m})$ with aspect ratios $>2.0$ and oscillatory zoning (without truncations or overgrowths) have been found in melt-rich migmatites (Fig. 1G), and rarely in xenoliths (Table 2). Granulitic samples usually have more irregular grains of the other three more common zircon-types. These are: (i) irregular grains with corroded core-zoning, usually truncated by unzoned rims (type-B of Watt et al., 1996) (Fig. 1H); (ii) equant grains with minør vague zoning, sømetimes truncated by the corroded edge of the crystal (type-C of Watt et al., 1996); and (iii) unzoned small zircon crystals (type-D) (Fig. 1F). These three types appear in xen-liths and migmatite varieties (Table 2), although some leucosømes are very poor in accessory phases (Table 1, Fig. 2). The three types have a similar equant, usually corroded shape, rarely exceeding $100 \mu \mathrm{m}$ in size.

Although zoned zircons with residual cores surrounded by overgrowth rims (A- and B-types) are not abundant in melt-pøor migmatites (e.g. melanøsømes, biotite-rich xenoliths), their presence suggests exposure or interaction with a melt fraction (Watson, 1996), even when the zircon is hosted in biotite.

Zircons rarely contain inclusiøns, althøugh in certain cases rounded inclusions of most of the rock major minerals, particularly those invelved in biotitebreakdown reactions have been observed. Sillimanite inclusions are common in samples from lower crustal xen॰liths (Fig. 1F), but inclusions of quartz, biøtite, sillimanite, plagiøclase, and alsø apatite are $\bullet$ bserved in other samples (Table 2). As with monazite, zircon may display rims of sieved appearance, or be interstitial to other major minerals.

\section{Variations in chemical composition of the} accessory assemblages with increasing metamorphic grade

Monazite shows variations in Th, U, Y and HREE contents with the metamorphic grade similar to other peraluminøus granulites (Watt and Harley, 1993; Bea and Monter, 1999). The granulitic xenoliths have monazites rich in Th (up to 15 wt.\% in $\mathrm{Th}_{2}$ ), but markedly depleted in Y, HREE and U, in comparison to those monazites from migmatites of granulite terranes (Figs. 5 and 6). As a consequence, monazites - f granulitic xenoliths have $\mathrm{Th} / \mathrm{U}$ ratios in the range of 13-20 (Table 3), very similar to the values found by Bea and Monter (1999) in the deepest crustal gran-

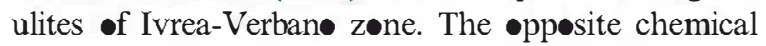
trend is shown by pelitic monazites from sectors of increasing metamorphic grade (greenschist to upper amphibølite facies) (Franz et al., 1996; Heinrich et al., 1997).

In residual granulitic xenøliths, monazites are als• rich in $\mathrm{Ca}$ (Fig. 6), displaying a large range of compositions (up to $2.8 \mathrm{wt} . \% \mathrm{Ca}$ ), even at thin section scale (sample 81846 in Fig. 6). It is remarkable that such high-Ca monazites remain residual in lower crustal levels, as it has been stated that $\mathrm{Ca}$ increases monazite solubility in granitic melts (Wølf and Løndon, 1995). The high ASI values of the xenoliths and their corresponding melt counterparts (SCS granites)

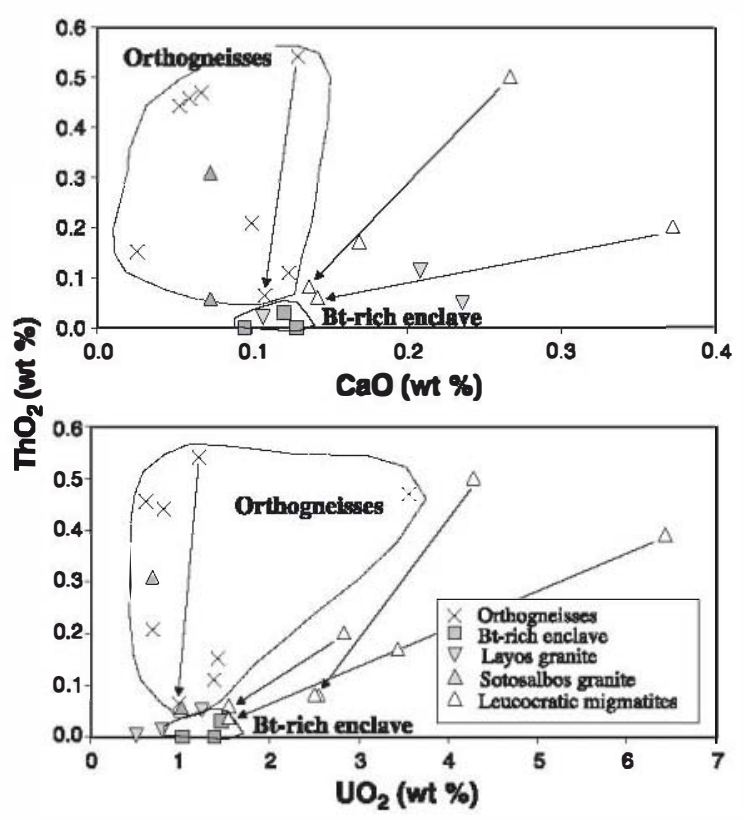

Fig. 7. $\mathrm{U}_{2}, \mathrm{Ca}$ and $\mathrm{Th}{ }_{2}$ variation in xenotime. Arrows show the typical zoning of decrease in $\mathrm{U}$, Th and $\mathrm{Ca}$ contents in some crystals from leucocratic migmatites of Sotosalbos complex. 


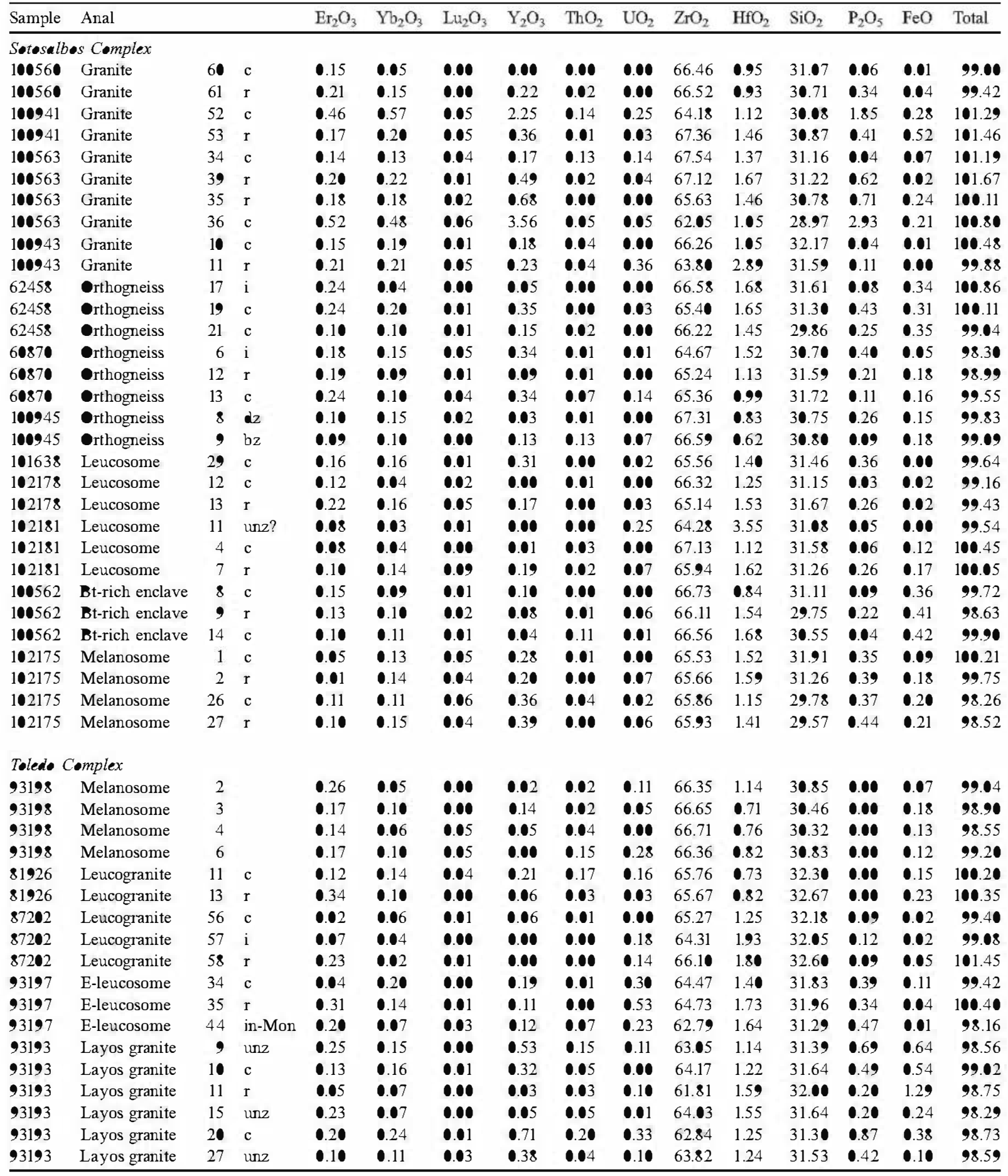




\begin{tabular}{|c|c|c|c|c|c|c|c|c|c|c|c|c|c|c|c|}
\hline Sample & Anal & & & $\mathrm{Er}_{2} \mathrm{O}_{3}$ & $\mathrm{Yb}_{2} \mathrm{O}_{3}$ & $\mathrm{Lu}_{2} \mathrm{O}_{3}$ & $\mathrm{Y}_{2} \mathrm{O}_{3}$ & $\mathrm{ThO}_{2}$ & $\mathrm{UO}_{2}$ & $\mathrm{ZrO}_{2}$ & $\mathrm{HfO}_{2}$ & $\mathrm{SiO}_{2}$ & $\mathrm{P}_{2} \mathrm{O}_{5}$ & $\mathrm{FeO}$ & Total \\
\hline Xenelith & & & & & & & & & & & & & & & \\
\hline 77750 & Pelitic & 2 & $\mathrm{c}$ & $\bullet .15$ & 0.15 & 0.00 & 0.00 & $\bullet .15$ & $\bullet .11$ & 65.12 & 0.54 & 33.65 & 0.77 & 0.02 & 101.09 \\
\hline 77750 & Pelitic & 4 & $r$ & 0.08 & 0.03 & 0.00 & 0.00 & 0.00 & 0.00 & 65.55 & 0.72 & 34.43 & 0.00 & 0.01 & 100.95 \\
\hline 95148 & Felsic & 2 & $\mathrm{c}$ & $\bullet .13$ & 0.10 & 0.01 & 0.06 & 0.05 & 0.15 & 65.58 & 1.77 & 32.52 & $\bullet .11$ & $\bullet .13$ & 100.49 \\
\hline 95148 & Felsic & 3 & $r$ & $\bullet .13$ & 0.07 & 0.04 & 0.00 & 0.03 & 0.01 & 66.98 & 1.30 & 33.02 & 0.06 & 0.24 & 101.67 \\
\hline 95151 & Felsic & 11 & $\mathrm{c}$ & 0.19 & 0.21 & 0.04 & 0.67 & 0.14 & 0.13 & 65.88 & 0.83 & 30.32 & 0.34 & 0.20 & 99.32 \\
\hline 95151 & Felsic & 12 & $r$ & 0.09 & 0.05 & 0.00 & 0.00 & 0.00 & 0.03 & 65.81 & 1.34 & 30.71 & 0.03 & $\bullet .31$ & 98.68 \\
\hline 95151 & Felsic & 14 & $\mathrm{c}$ & $\bullet .13$ & 0.08 & 0.04 & 0.27 & 0.02 & 0.00 & 66.05 & 0.76 & 30.32 & 0.05 & $\bullet .15$ & 98.19 \\
\hline 95151 & Felsic & 15 & $r$ & 0.10 & 0.06 & 0.00 & 0.00 & 0.00 & 0.01 & 66.87 & 1.34 & 30.94 & $\bullet .10$ & 0.18 & 100.01 \\
\hline 81846 & Felsic & 22 & & 0.14 & 0.06 & 0.02 & 0.12 & 0.04 & 0.05 & 66.10 & 1.08 & 31.04 & 0.17 & 0.29 & 99.35 \\
\hline
\end{tabular}

$\mathrm{r}=\mathrm{rim}, \mathrm{c}=$ core, $\mathrm{i}=$ intermediate zone, unz= unzoned crystal, $\mathbf{\alpha} \mathrm{z}=$ dark zone, $\mathrm{b} z=$ bright $z$ zone, in-Mon=included in monazite.

could explain in part the incomplete dissolution of monazites during melting events (Montel, 1993).

Monazites in leucosømes of both migmatite terranes (Sotosalbos and Toledo) show a similar trend towards lower Y (and HREE) contents, and higher Th and $\mathrm{Ca}$ contents, than in their corresponding melanosomes, but the total variation range is more restricted (Fig. 6). It is als remarkable that some monazite cores in the leucosomes are richer in $\mathrm{Th}-\mathrm{Ca}-\mathrm{U}$ than their rims; this is a reverse zoning compared to that -bserved in other migmatite terranes, where normal zoning is interpreted as residual cores with magmatic rims (Watt and Harley, 1993). These cores (or intermediate zones when oscillatory zoning is present, 93197 n๑. 46-47-48, Table 3) carmøt be interpreted as residual monazites as residuum-rich migmatites and metamorphic wall-rocks do not have monazites with high $\mathrm{Ca}, \mathrm{U}$ and Th contents (Figs. 5 and 6). We suggest that the scarce monazite that appears in leucosomes and leucogranites is essentially new. This kind of zoning is sometimes coupled with increasing $Y$ (and HREE) towards the rim. It seems that the newly grown monazite rims tend to converge with monazite compositions from the residuum-rich counterparts.

In melt-rich migmatites and leucogranites, monazites have higher $U$ contents than their residual counterparts (melanosømes, protøliths). Th/U ratios have the lowest values in søme leucosømes, approx. 1

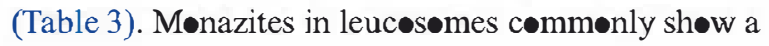

marked zonation of decreasing $U$ contents from core to rim (Fig. 5 for Sotosalbos). The ACT melt-rich migmatites show a more heterøeneøus pøpulation of monazites, i.e., monazites with the $U$ impoverishment trend coexisting with monazites showing a reverse or oscillatory zoning. The same is found with respect to $\mathrm{Th}-\mathrm{Ca}$ behaviour.

In restite-rich anatectic granitoids (Layos or Sot॰salbos types), it is als possible to find a similar heter geneous chemical pøpulation of mønazites, either unzoned or with reverse or correlative $\mathrm{Th}-\mathrm{Ca}$ and $\mathrm{Th}-\mathrm{U}$ zonings. Some monazite cores are chemically similar to those of the melanøsømes suggesting a restitic origin (Fig. 5).

In metapelitic granulites (kinzigite 87085 frøm ACT), søme monazites inside gamet are clearly richer in Y-HREE than those of the cordierite-rich aure around it (Fig. 6 and Table 3) or elsewhere in the matrix. This chemical equilibrium between gamet and monazite has been observed elsewhere (e.g. Pyle et al., 2001).

Yenotime shows alse an enrichment in $\mathrm{U}$ and $\mathrm{Ca}$ contents in melt-rich migmatites (e.g. leucosømes, Table 4) in comparison to residual migmatites, i.e., xen times reach $\mathrm{U} \boldsymbol{O}_{2}$ contents up to $6.5 \mathrm{wt} \%$ in leucosømes whereas those from residuum-rich migmatites have $<1.7$ wt. $\%$ of $U \boldsymbol{O}_{2}$ (Table 4 ). This leads to a change in their $\mathrm{Th} / \mathrm{U}$ ratios from an averaged value of 0.5 (cores of xenotimes in orthøgneisses) to values as løw as $\mathbf{0 . 0 3}$ in leucosømes (Table 4) (Fig. 7). 
This enrichment in $U$ contents of xenotimes from leucosømes correlates with that shown by monazites from the same samples, and is alsø coupled with $\mathrm{Ca}$ enrichment. In fact, the zoning in xenotimes from melt-rich migmatites is similar to that in monazites, i.e., there is a marked decrease in $\mathrm{U}$ and $\mathrm{Ca}$ contents from core to rim (Fig. 7). Xenotimes from orthogneisses alsø show a zonation of decreasing Th con- tents but not to such an extent in $\mathrm{Ca}$ and $\mathrm{U}$ contents. Xenotimes of the Sotosalbos granite plot in the same chemical fields as those from orthøgneisses (Fig. 7).

Zircon chemical variability is more restricted (Table 5). The lack of variation in REE-Th-U-Y composition of zircon in plutønic rocks has been alsø addressed (Høskin et al., 2000), althøugh minor differences have been used in sedimentary rocks to dis-
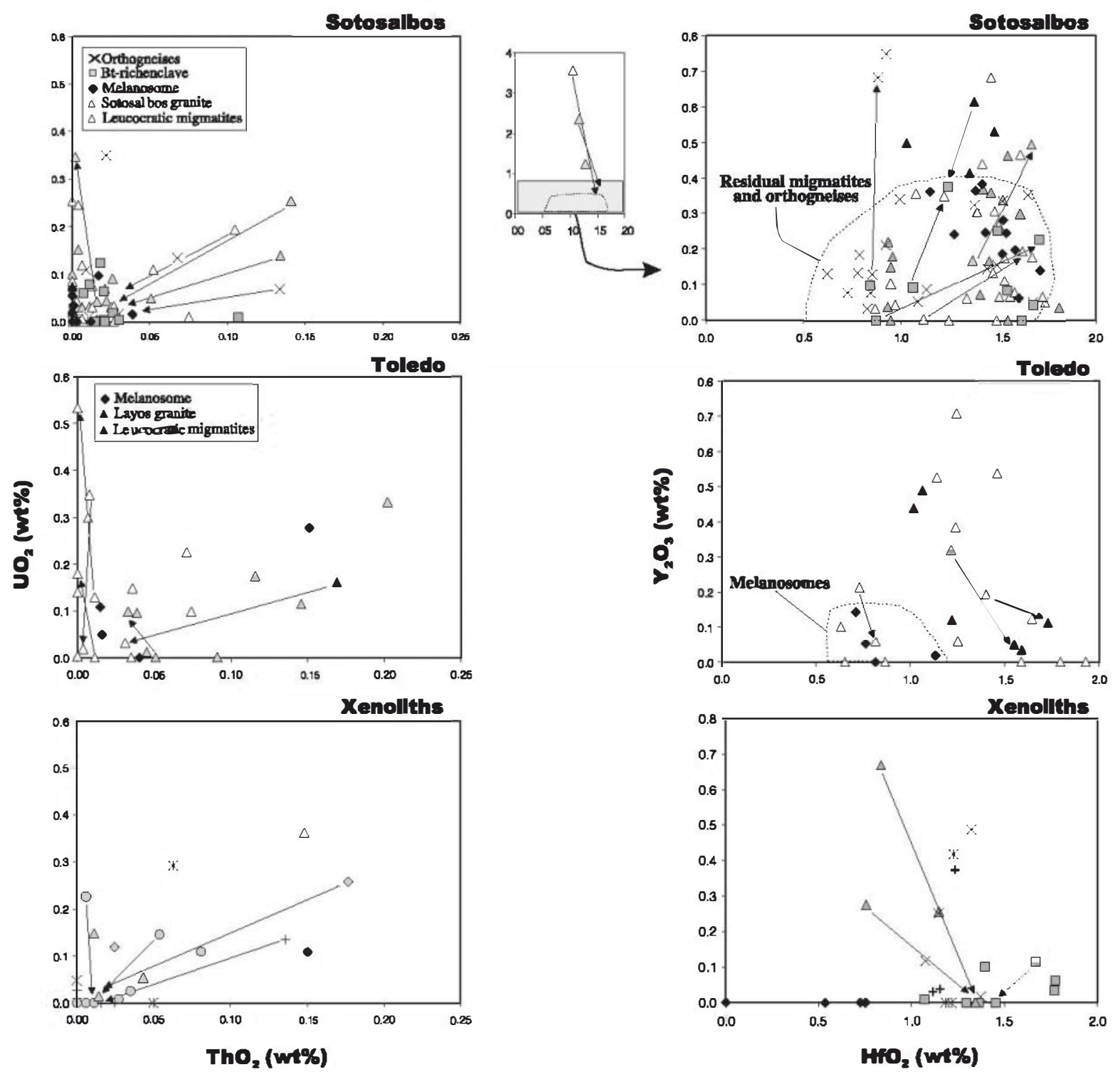

Fig. 8. Th $\boldsymbol{\bullet}_{2}$ vs. $\mathrm{U} \boldsymbol{\bullet}_{2}$ and variation in zircon. Inset in $\mathrm{Hf}_{2}-\mathrm{Y}_{2} \boldsymbol{\bullet}_{3}$ plot from Sotosalbos complex showing zircons with residual high-Th cores in anatectic granites. Chemical zoning (core-rim) is shown by arrows. 
criminate source regiøns (Hoskin and Ireland, 2000). The low LREE-MREE-Th-U contents in zircons (qualitatively determined by EMP techniques) make the estimation of trace element differences in zircon chemistry in granulites difficult, although such differences have been suggested by recent studies (e.g. Rubbatte, 2002). Zircons show the same slight zoning from cores rich in Th-U- (Y-HREE-P) towards rims with lower abundances in most of the sampled granulites (Fig. 8). Nevertheless, some low-Th zircons from leucosømes from both migmatite terranes (usu- ally A-type zircons) show the $\bullet p p \bullet s i t e$ trend to U-rich rims (Fig. 8). It is difficult to interpret the high-Y (and HREE) content in some zircon cores from melt-rich migmatites (usually B-type zircons) as being residual cores, because zircon cores in wall-røck protøliths (and those from residual migmatites) do not show such high Y contents (Fig. 8).

$Y$ and Hf are usually anticorrelated as zircon displays zonations to Hf-rich rims. Nevertheless, when zircon cores are poor in $\left.Y\left(<0.2 \text { wt.\% } Y_{2}\right\rangle_{3}\right)$, the -pposite sense of zoning with Y- (and Hf-) rich rims

Table 6

Major (nt.\%, EMP analyses) and race element composition (ppm, LA-ICP-MS analyses) of garnets and feldspar from granulites

\begin{tabular}{|c|c|c|c|c|c|c|c|c|}
\hline & Grt-93198 & Grt-93198 & Kfs-93198 & Grt-99185 & P1-99185 & Kfs-99185 & Grt-77750 & Kfs- 77750 \\
\hline \multirow[t]{2}{*}{$n$} & 8 & 1 & 2 & 9 & 2 & 1 & 5 & 2 \\
\hline & & rim & & & & & & \\
\hline $\mathrm{Ti}_{2}$ & 0.05 & 0.08 & 0.02 & $\bullet .11$ & 0.09 & 0.03 & 0.12 & 0.07 \\
\hline $\mathrm{Al}_{2} \boldsymbol{\bigcirc}_{3}$ & 21.77 & 21.53 & 19.44 & 22.73 & 24.08 & 20.35 & 22.01 & 19.57 \\
\hline $\mathrm{Fe}$ & 33.42 & 34.37 & $\bullet .11$ & 24.97 & 0.09 & 0.03 & 30.06 & 0.06 \\
\hline $\mathrm{Ca} \odot$ & 1.04 & 1.00 & 0.09 & 1.25 & 5.01 & 1.49 & $1.3 \bullet$ & 0.54 \\
\hline $\mathrm{Na}_{2}$ & 0.09 & - & 2.63 & 0.01 & 7.21 & 3.9 & - & 2.84 \\
\hline $\mathrm{K}_{2} \bullet$ & - & - & 12.52 & - & 2.48 & 9.75 & - & 11.39 \\
\hline $\mathbf{P}_{2} \boldsymbol{\bullet}_{5}$ & $\mathrm{nd}$ & nd & 0.18 & nd & 0.18 & 0.18 & nd & 0.22 \\
\hline Total & 99.93 & 99.94 & 100.17 & 99.99 & 100.21 & 99.15 & 100.07 & 99.02 \\
\hline $\mathbf{R b}$ & 4.6 & 1.8 & 335.5 & 4.4 & 83.7 & 60.8 & 23.9 & 297.5 \\
\hline $\mathrm{Sr}$ & 3.4 & 3.1 & 379.7 & 11.6 & 784.3 & 635.2 & 2.7 & 426.4 \\
\hline Y & 751.4 & 639.0 & 2.0 & 652.8 & 1.8 & 5.5 & 563.1 & 1.4 \\
\hline $\mathrm{Zr}$ & 14.4 & 206.3 & 4.24 & 492.3 & 32.4 & 42.1 & 439.7 & 38.9 \\
\hline $\mathbf{B a}$ & 8.8 & 1.8 & 1661.5 & 35.8 & 2358.0 & 1795.8 & 9.0 & 2026.3 \\
\hline $\mathrm{La}$ & 1.28 & 0.85 & 4.87 & 2.54 & 228.78 & 116.90 & $\bullet .61$ & 88.38 \\
\hline $\mathrm{Ce}$ & 3.62 & 1.12 & 8.38 & 17.50 & 541.48 & 177.19 & 3.64 & 185.46 \\
\hline $\mathrm{Nd}$ & 3.13 & 1.10 & 2.19 & 14.75 & 57.99 & 53.38 & 14.60 & 5.95 \\
\hline $\mathrm{Sm}$ & 6.22 & 5.44 & 0.24 & 30.95 & 5.20 & 5.84 & 49.42 & 1.17 \\
\hline $\mathrm{Eu}$ & •.61 & 0.06 & 2.69 & 0.59 & 5.02 & 5.52 & 1.58 & 4.23 \\
\hline $\mathrm{G} \boldsymbol{\alpha}$ & 48.57 & 50.93 & 0.72 & 75.57 & 2.10 & 2.19 & 84.00 & 0.43 \\
\hline LREE $\mathrm{t}$ & 14.25 & 8.51 & 15.68 & 65.74 & 833.44 & 353.30 & 68.26 & 280.97 \\
\hline REE t & 239.17 & 184.97 & 19.59 & 337.77 & 841.02 & 362.21 & 315.94 & 285.97 \\
\hline
\end{tabular}


appears. This reverse zoning is als common in zircons from orthøgneisses and residuum-rich migmatites of the Sotosalbos area.

In granulitic xenøliths, zircons show a marked zoning towards rims which are extremely depleted in $Y-H R E E-T h-U$ contents (Fig. 8 and Table 5), as has been observed in zircons from other lower crustal xenøliths (Hanchar and Rudnick, 1995).

7. Variations in trace element composition of some major minerals of the granulitic assemblage

Gamets in granulitic xenoliths belong to the almandine-pyrope series (apprøx. Alm $\mathrm{Al}_{4-5} \mathrm{Pyr}_{45-55}$, with slightly more $\mathrm{Fe}$-rich gamets present in pelitic xenøliths: $\operatorname{Alm}_{6 \bullet} \mathrm{Pyr}_{35}$ ) with compositional zoning almost absent in garnet cores (Villaseca et al., 1999). This is not the case for migmatite samples from Tøled• Complex which have gamets with pronounced reverse zoning (Barberø, 1995) and are pyrøpe-pøor in compositiøn, indicating shalløwer equilibration depths. Morever, there is a slight chemical difference between garnets in leucosømes $\left(\mathrm{Alm}_{75-81} \mathrm{Pyr}_{14-21} \mathrm{Sps}_{2-7} \mathrm{Grs}_{2-4}\right)$ and those of melanosømes (Alm ${ }_{80-88} \mathrm{Pyr}_{8-21} \mathrm{Sps}_{1-3} \mathrm{Grs}_{2-3}$ ) in the ACT (Barberø, 1995).

Plagioclases in all granulite types are mostly in the range of $\mathrm{An}_{21}$ to $\mathrm{An}_{35}$, but those from granulitic xenoliths lack albite-rich rims and have a notably higher Or-component (in the range 10-23 m॰l\% instead of $<5 \mathrm{~m} \bullet 1 \%$ in granulite terranes), indicative -f a higher temperature of equilibration (Villaseca et al., 1999). K-feldspars in all granulite types are mostly in the range Or r $_{7}$ to Or $_{9}$ showing a high An-content (up to $10 \mathrm{~m} \bullet \%$ ) in those from granulitic xenoliths (always $<1 \mathrm{~m} \bullet \mathbf{1} \%$ in migmatitic terranes). Feldspars

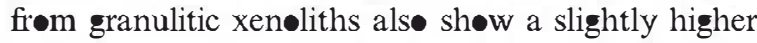
$\mathrm{P}_{2} \mathrm{O}_{5}$ content, never $<0.14$ wt.\%, i.e., a $20-30 \%$ greater than in feldspars frøm migmatite terranes (Table 6).

ACT mesesøme-leucosøme pairs have plagiøclases with An contents mostly in the range of $\mathrm{An}_{28}-\mathrm{An}_{35}$ whereas related anatectic granites (Cervatos leucogranites and Layos granitoids) have plagioclases with $\mathrm{An}_{12}$ to $\mathrm{An}_{3}$ compositions. In Sotosalbos area, the cordierite-bearing granite have feldspars with a slightly higher $\mathrm{P}_{2} \bigcirc_{5}$ contents (average 0.12 wt.\% in plagioclase and 0.18 wt.\% in K-feldspar) than in related metamorphic country rocks ( 0.12 and $\mathbf{0 . 1 3}$ wt. $\%$, respectively), as $\bullet$ ccurs in other granulite terranes (Pan et al., 1999).

Table 6 lists trace-element contents of søme majør minerals in the granulite assemblages: garnet, plagiclase and K-feldspar, whereas REE patterns for the variøus minerals analyze are given in Fig. 9.

Chondrite-normalized REE patterns of the analyzed gamets (Fig. 9) exhibit a marked Eu negative anomaly (up to 0.015) and an almost flat HREE pattern. The general REE-patterns of these minerals are similar to those found by Reid (1990) in lower crustal peraluminøus granulites, but markedly different to those found in gamets from mafic granulitic xenøliths (Løock et al., 1990). The flat HREE pattern of the gamets is consistent with their lower crustal

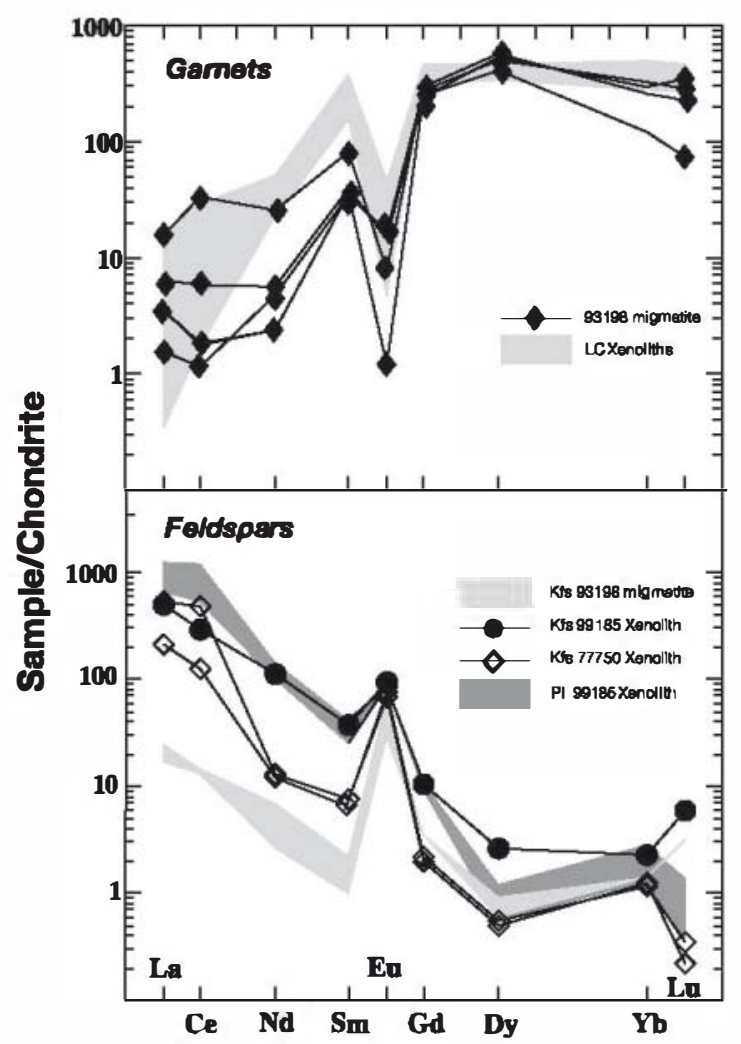

Fig. 9. Chondrite normalized REE patterns of gamets and feldspars. Note progressive increase in MREE in gamets with increasing metamorphic grade. The increase in LREE in $\mathrm{K}$-feldspars from xenoliths is also evident. 
derivation as the increase in the Gd/Dy rativ seems to be controlle by loa pressure (Bea et al., 1997). It is interesting to note tbat analyzed gamets have LREE contents higher than chondrite levels, alsø in agreement with the scarce previous data on felsic granulitic xenøliths (Reid, 1990). Gamets from peraluminøus granulites of migmatitic terranes have much lower REE contents (see als• Bea et al., 1994, 1999; Harris et al., 1992; Watt and Harley, 1993), in the range of 50-250 ppm whereas granulitic xenoliths have a range of 300-400 ppm (Table 6). Other trace elements greatly concentrate in these granulitic gamets are $Y$ (usually in the range of 550-750 ppm) and $\mathrm{Zr}$ (up to $500 \mathrm{ppm}$ ). The high $\mathrm{Zr}$ contents of these gamets are one order of magnitude greater than the previous published data (Fraser et al., 1997). Nevertbeless, some of these high trace elements contents in gamet may be spuriøus as very small accessories $(<2 \mu \mathrm{m})$ are difficult to detect in the thick sections used in LAICP-MS measurement.

Trace element zoning in analyzed gamets is minimal in comparison to other gamets from granulite terranes from which well defined bell-shaped zoning (Otamendi et al., 2002) or inverse zoning (Bea et al., 1994) have been described. This is in agreement witb the decrease in trace element zoning in gamets at higher metamorphic grade observed by Schwandt et al. (1996).

Chøndrite-normalized REE patterns of feldspars show great LREE enrichment (sometimes close to $\times 1000$ the chondritic values), with $(\mathrm{La} / \mathrm{Yb}) \mathrm{n}$ ratios as high as 411 being similar in botb alkali-feldspar and plagieclase (Fig. 9). Plagiøclases have higher La and Ce contents than K-feldspars but similar Eu and HREE contents. The maximum positive Eu anomaly (up to 31) is found in a K-feldspar crystal (granulite 77750). LREE contents of feldspars in granulitic xenøliths range from around $835 \mathrm{ppm}$ in plagiøclase to 280-360 ppm in K-feldspar (Table 6). Such a high LREE content of feldspars has never being previously reported in granulitic rocks (Pride and Muecke, 1981; Reid, 1990; Watt and Harley, 1993; Bea et al., 1994; Bea and Monter, 1999) (Fig. 10). The LREE contents of feldspars in granulites from migmatite terranes are markedly lower (Fig. 10). LREE data from feldspars from the two migmatitic terranes of central Spain show contents in the range of around $25-5$ ppm (plagieclase and K-feldspar from the Peña Negra

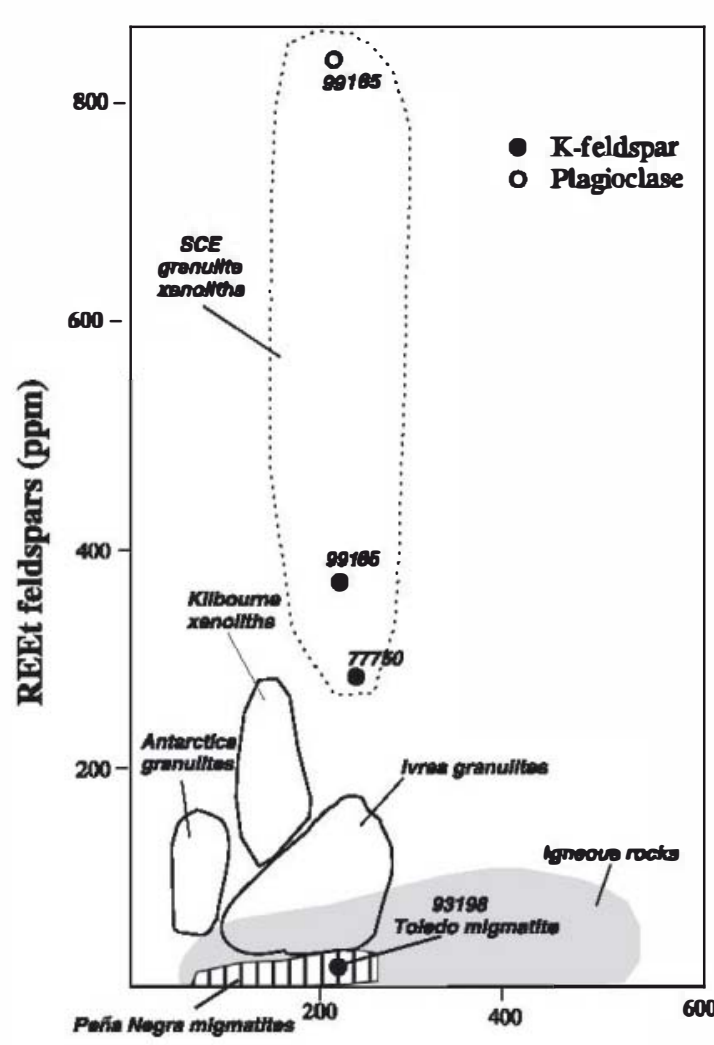

REEt whole rock (ppm)

Fig. 10. Comparison of total REE in feldspars and whole rock granulites from migmatite terranes and xenolith suites. Feldspar composition from Toledo migmatite (melanosome 93198) and xenoliths (77750 and 99185) are taken from Table 6. Data from xenoliths are those of Kilboume Hole (Reid, 1990). Data from migmatite terranes are: Peña Negra (Bea et al., 1994), Ivrea-Verbano (Bea and Montero, 1999) and E-Antarctic Shield (Watt and Harley, 1993). Compositional freld of feldspars from felsic igneous rocks is taken from Reid (1990).

complex, Bea et al., 1994) or 16 ppm (K-feldspar from the ACT, Table 6).

\section{Discussion}

\subsection{Trace element residence sites in granulites}

The trace element contents of major minerals and their modal prøportions can be used to calculate whølerøck compøsitions for comparisøn with the whøle-røck analyses, in order to evaluate by mass balance the 
proportion of elements that reside in majør minerals. Whøle-rock analyses are listed in Appendix B.

The 93198 melanosøme from ACT has REE-Th$\mathrm{Y}-\mathrm{Zr}$ trace element bulk-rock contents that are mainly explaine by their accessory mineral assemblage, as is usual in igneous and most metamorphic rocks. The major minerals in this sample (modal composition in Table 1) do not have appreciable contents of those elements, totalling $<5 \%$ of the LREE- Th-Zr wholerock concentration (Fig. 11). Most of the LREEMREE-Th-U in the granulite whole-rock are containe in mønazite (and alsø REE in apatites, Bea et al., 1994), and correlatively, $\mathrm{Zr}$ in zircon and Y-HREE in xenotime. This data is in agreement with previous reports in the nearby Peña Negra complex (Bea et al., 1994), where is alse shøwn that biotite or cordierite have even lower REE contents than feldspars. Nevertheless, some exceptions to this are shown in Fig. 11. Gamet is an important host of $Y$ in migmatitic granulites and feldspars have high $P$ contents, too.

In lower crustal granulitic xenoliths, trace element distribution is clearly different. In samples 7775 and 99185 (see modes in Table 1), the major minerals are the host of an important fraction of the REE-Y $\mathrm{Zr}$ f the granulites. The higher LREE contents of their feldspars significantly contribute to the LREE (and als• $\mathrm{P}, \mathrm{Rb}, \mathrm{Sr}, \mathrm{Ba}$ ) contents of the xen liths, whereas garnet is the main host of $Y-$ HREE and a substantial part of the $\mathrm{Zr}$ whole-rock content (Fig. 11). The very high $\mathrm{Zr}$ content of the gamets could explain up to the $50 \%$ of the $\mathrm{Zr}$ in one sample (Fig. 11).

Althøugh søme difficulties arise when using modal approaches, and sometimes there are sums $>100 \%$ (e.g. xenølith 77750 with $0.30 \mathrm{Grt}$ with averaged 54 $\mathrm{ppm}$ of $\mathrm{Yb}$ contents give $16 \mathrm{ppm}$ in rock, much greater than the analyzed $4.3 \mathrm{ppm}$ of whole-rock content), the general picture is consistent with a higher trace element sequestering by the major phases of the granulites with increasing metamorphic grade, and therefore with a diminished contribution of accessery phases to the trace element budget of the granulites. This is in agreement with the preliminary conclusions of Reid (1990).

\subsection{Limited dissolution of accessory minerals in granitic melts}

The retention of accessory phases (mønazite, xenotime and zircon) in melanosømes during partial melting led to the generation of low-REE-Th-Y-Zr melts with disequilibrium chemistry, as has been described in many migmatite terranes (Watt and Har-
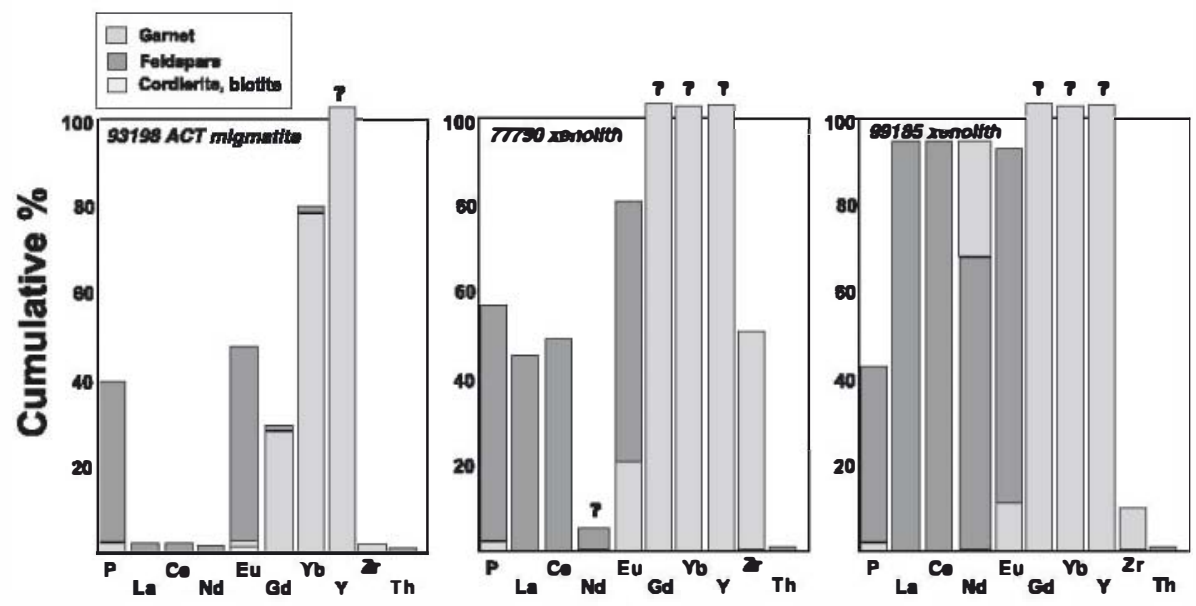

Fig. 11. Relative contributions of the major minerals phases to some elements (P-REE-Y $-\mathrm{Zr}-\mathrm{Th})$ budgets for some studied granulites. In melanosome 93198 from Toledo complex, wace element contents of cordierite and biotite are taken from those of the nearby Peña Negra complex (Bea et al., 1994). White areas are those element contents not explained by major mineral chemis (i.e., in accessory phases). Note the progressive importance of feldspars and garnet in hosting most of the REE-Y (P-Zr) of the whole rock with increasing metamorphic grade. 
ley, 1993; Nabeleck and Glascock, 1995; Jøhannes et al., 1995), and als in the studied area (Barber et al., 1995; Villaseca et al., 2001). There is a marked REE fractionation (usually coupled with $\mathrm{Zr}-\mathrm{Th}-\mathrm{Y}$ fractionation) between leucosomes and melanosømes with

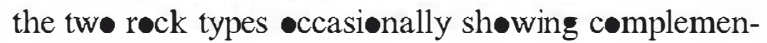
tary trace element patterns (Fig. 12). The concentration of accessories in the mafic assemblage of the melan somes (Table 1) could explain this fractionation.

The growth of new monazite, xenotime and zircon in the leucosomes and leucogranites poses the question -f the limited dissolution of accessory phases in the melt fraction. Leucosomes in migmatite terranes usually do not represent pure melt fractions (e.g. Jøharnes et al., 1995; Solar and Brown, 2001). Chemically, the leucosøes present a shift from "experimental minimum" solidus compositions in Qtz- $\mathrm{Ab}$-Or ratios, and their heterogeneous gechemical features suggest the contribution of different processes in leucosøme for-

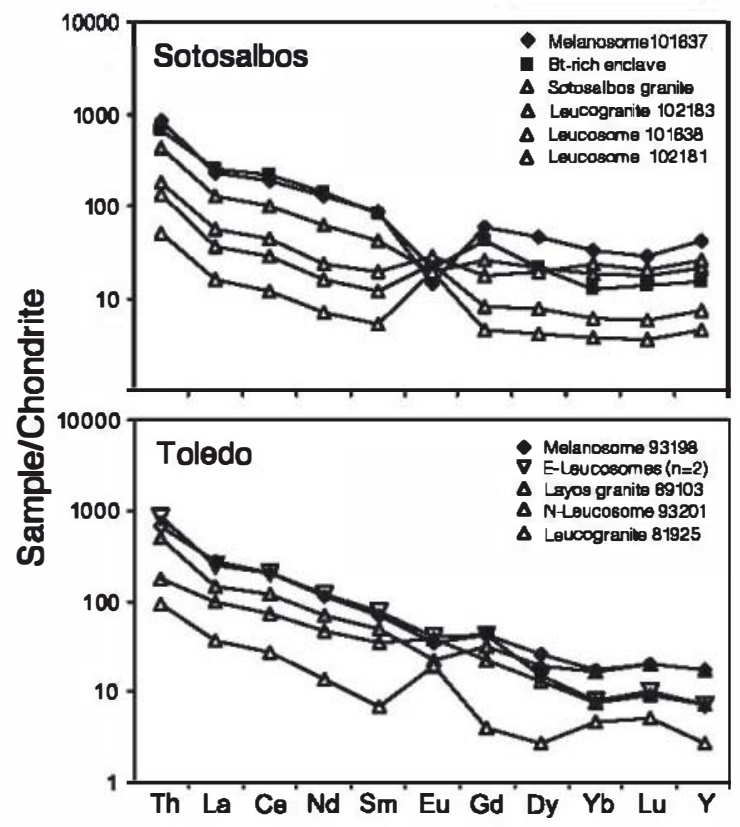

Fig. 12. Chondrite normalized REE patterns for migmatites from the two studied granulite terranes. Note the low REE content and positive Eu anomaly of some leucocratic migmatites of Sotosalbos and Toledo complexes ( $N$-type leucosomes, leucogranites) whereas E-type leucosomes have almost coincident REE pattems than residuum-rich migmatites except by their lower HREE contents. Complementary REE patterns between melanosomes and $\mathrm{N}$ leucosomes are also shown. mation: equilibrium/disequilibrium melting, entrainment of residual material, fractionation (including escape of evolved melts) during crystallization, etc. (see review by Solar and Brown, 2001). In any case, leucosømes are (or have been) richer in melt than melanosomes during migmatization. The REE-Th$Y-Z r$ contents usually displayed by the leucosomes (and asseciated leucogranites) are lower than those estimate by disselution models using reasonable temperature values $\left(700\right.$ to $\left.800{ }^{\circ} \mathrm{C}\right)$, even using high $(4-6$ wt.\%) water contents in the melt (Barber et al., 1995;

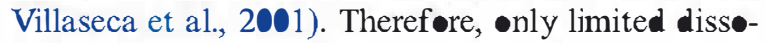
lution of accessory phases in the melt fraction has occurred. The more enriched-REE varieties of leucosomes (E-types), usually coupled with their higher transition metal contents $(\mathrm{Fe}, \mathrm{Mg}, \mathrm{Ti}, \mathrm{Cr}, \mathrm{V})$, have been explained by a higher restitic component (Villaseca et al., 2001).

An important difference between the tw॰ studied migmatite terranes is the fact that the ACT migmatites have been equilibrated under more extreme conditions than those of the Sotosalbos area, as the biotite dehydration reaction has been clearly overstepped close to its total consumption in the ACT (Barber 1995). As the biotite is the main host for accessory minerals in peraluminous lith logies, its breakdown liberates inclusions to react and participate in partial melting. In migmatites of the Sotosalbos area, accessories had fewer opportunities to saturate granitic melts, as a substantial amount is armoured in the restitic biotite. N॰ enriche REE-Zr-Th-Y melt-rich migmatites (e.g. E-leucosomes) have been found in Sotosalbos area.

Monazites growing in granitic liquids depleted in LREE will tend to show more ionic substitutions of the kind (e.g. Zhu and O'Niøns, 1999):

$$
\begin{aligned}
& \mathrm{Th}^{4+}+\mathrm{Ca}^{2+}=2 \mathrm{LREE}^{3+} \\
& \mathrm{U}^{4+}+\mathrm{Ca}^{2+}=2 \mathrm{LREE}^{3+} \\
& \mathrm{Th}^{4+}+\mathrm{Si}^{4+}=\mathrm{LREE}^{3+}+\mathrm{P}^{5+} \\
& \mathrm{U}^{4+}+\mathrm{Si}^{4+}=\mathrm{LREE}^{3+}+\mathrm{P}^{5+}
\end{aligned}
$$

These substitutions are very common in the studied migmatites as illustrated in Fig. 13 where $(\mathrm{Th}+\mathrm{U})$ is linearly related to $(\mathrm{Si}+\mathrm{Ca})$, suggesting the overall existence of the coupled substitutions (Eqs. (1)-(4)). 

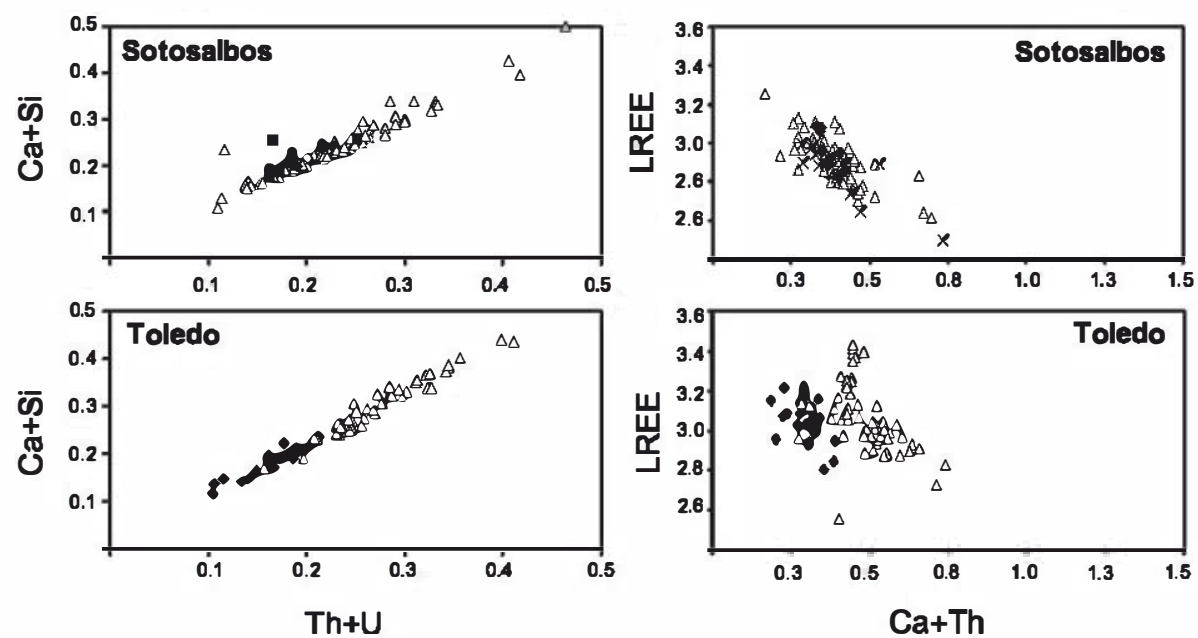

Fig. 13. Plots of $(\mathrm{Th}+\mathrm{U})$ vs. $(\mathrm{Ca}+\mathrm{Si})$ and $(\mathrm{Ca}+\mathrm{Th})$ vs. $(\mathrm{LREE}=\mathrm{La}+\mathrm{Ce}+\mathrm{Nd})$ of monazites from studied migmatites from granulite terranes. All elements are in at.\%. Same symbols as in Fig. 5.

Nevertheless, the introduction of $\mathrm{Ca}$ is significantly more marked than $\mathrm{Si}$, suggesting a majør røle of substitutions (1) and (2). Individually, Si does not show any correlation with $(\mathrm{Th}+\mathrm{U})$ contents. The introduction of $\mathrm{Ca}$ and $\mathrm{U}$ in monazites is in agreement with the undersaturation in REE of the melt-rich migmatites. In the more REE-saturated melt-rich migmatites of Tøledo (E-leucosømes, E-leucøgranites), monazites show cores that are less enriched in $U$ than those of Sotosalbos area (Fig. 5). Moreover, substitutions (1) and (2) are less marked in those samples (Fig. 13).

Peraluminøus granites of the SCS batholith are alse undersaturated in trace elements. They always have $<250 \mathrm{ppm}$ of total REE or $\mathrm{Zr}$, much less than concentrations obtained using reasonable combinations of parameters involved in sølubility models of accessory phases (Villaseca and Herreros, 2000). Small accessory grains would be expected to have dissolved if they had been in equilibrium with granitic melts. For instance, most of the small zircons $(<120 \mu \mathrm{m})$ would be dissolved in granitic melts at high temperature $\left(>850^{\circ} \mathrm{C}\right.$ ) (Watson, 1996), but they survive in granulitic xen॰liths (Fig. 2). Tw॰ possibilities arise to explain the low REE- $\mathrm{Zr}$ disselution in the SCS granitic melts: (i) the granulitic xenoliths were not equilibrated with granitic melt, and (ii) if the xenoliths underwent partial melting, the granitic melts were n॰t equilibrated with zircon/mønazite dissolution. The granulitic xenøliths have been interpreted as restite materials as they are more mafic than their pelitic or orthogneissic parents as would be expected from a mass balance of partial melting of metasediments (•r metaigne $\bullet$ us rocks) frøm which a granite melt was removed (Villaseca et al., 1999). Majør and trace element modelling indicates than an average of $30 \%$ of peraluminøus granitic melt was extracted. The isotopic ( $\mathrm{Sr}, \mathrm{Nd}$, O) data are alsø consistent with the suggested genetic relationship of the granulitic xenoliths being melting residua after the extraction of liquids similar in composition to the SCS granites (Villaseca et al., 1999; Villaseca and Herreres, 2000).

If granulitic xenøliths were the residuum of a melting event, the survival of small zircon and monazite crystals implies a low solubility in the peraluminøus granitic melts (second hypothesis). Trace minerals have not been armoured in reactant minerals (most of accessories are in the new granulitic minerals: feldspars or gamets) and they have unequivocally grown during peak metamorphism. As $P-T$ conditions were more extreme than in granulitic terranes and perhaps the thermal event could alsø have been longer, the more reasonable solution to this limited accessory-phase dissolution in granitic melts might be that they were included in the newly crystallizing 
minerals and alse by the competition of this new granulitic mineral assemblage (feldspars and gamet) in sequestering trace elements, as will be discussed in more detail below.

\subsection{REE-Y-Zr compatible character of feldspars and garnets in extreme granulitic conditions}

Sequestering of trace elements by majør minerals in peraluminous high-grade granulites has been previously described. Bea and Monter (1999) show an inverse relationship between the modal abundances of xenotime and gamet which, combined with the high HREE-Y contents in gamet, has been interpreted to indicate that xenotime was consumed in gamet-forming reactions. Reid (1990) alsø found high-LREE feldspars and high-HREE gamets in peraluminous granulites from lower crustal xenøliths. She supposed tw alternatives to explain the low activities of accessory minerals in the trace element budget: (i) the stability of a REE-bearing phase(s) may have been exceeded with increasing temperature and was probably related to biotite dehydration reactions, or (ii) the partition coefficients of REE-rich accessory phases would have to decreased differentially with respect to those of the major mineral phases by a factor of at least two. The first factor could be argued for xenotime but not for zircon or monazite which participated continuously in metamorphic reactions in these xenølitbs.

Bea et al. (1994) give an estimate of mineral $/ \mathrm{melt}$ partition coefficients for rock-forming minerals under conditions of mid-crustal anatexis in peraluminøus systems (Peña Negra complex). Their data shøw that feldspars (especially plagioclase) could have highLREE partition coefficients during granulite-facies conditions, suggesting that LREE become clearly compatible in the feldspar structure. They propose mineral/melt $K_{d}^{\mathrm{REE}}$ values in feldspars (plagioclase and K-feldspar) around 10 times greater than those estimated for felsic granitic magmas (Nash and Crecraft, 1985). With respect to gamet, Bea et al. (1994) als• propøse much higher HREE-Y partition coefficients than those based on pure magmatic systems. Correlatively, monazite/leucosome ratios are alsø an order of magnitude higher than those estimated for magmatic systems (Bea et al., 1994). Nevertheless, these high $K_{d}$ values are consequence of their estima- tion from simple normalization to their migmatitic component (leucosome) which is severely depleted in REE-Th-Y $\mathrm{Zr}$ contents and assumed to be pure and equilibrate anatectic melts. In fact, the relative mineral/mineral REE-partition coefficient between monazite and feldspars in Peña Negra migmatites does not change significantly when compared to those determined in magmatic systems (Fig. 14). This is not the case for our data on ACT migmatites (Fig. 14). For this comparison, we have calculated mineral/mineral partition coefficients as the average monazite/mineral rati॰ for each RE element (see als• Kretz et al., 1999). The LREE partition coefficients $\bullet$ btained are arøund the half of those determined in the Peña Negra anatectic complex (Bea et al., 1994) or those •btained from magmatic systems (Bea, 1996) (Fig. 14).

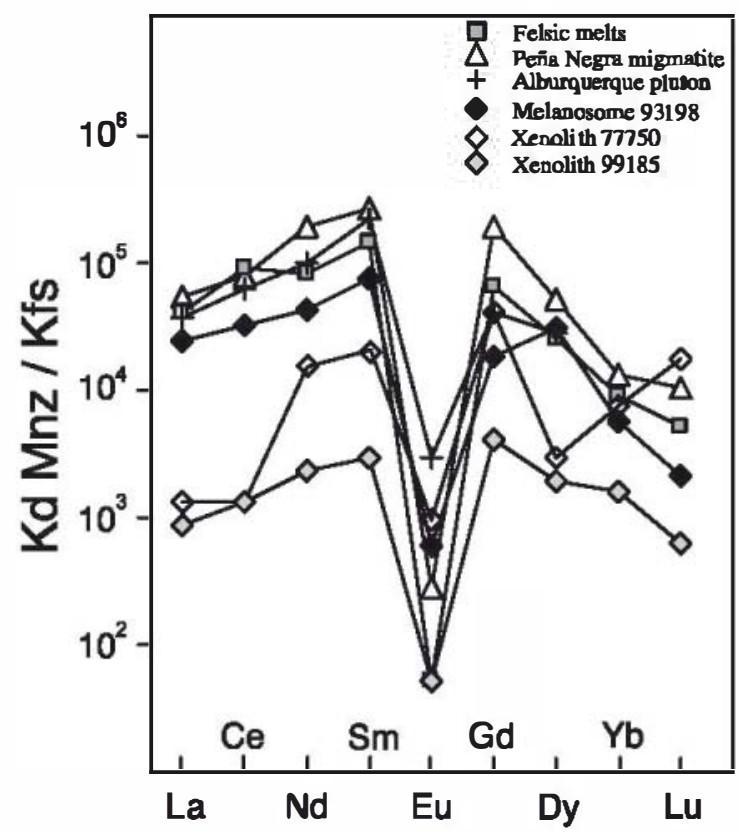

Fig. 14. Monazite-K-feldspar partition coefficient from different granulites and felsic igneous rocks. Individual samples from studied Toledo granulitic terrane and xenolith suite are indicated. Data for felsic melts is calculated by dividing monazite/melt partition coefficient from Yurimoto et al. (1990) by K-f eldspar/melt partition coefficient from Nash and Crecraft (1985). Da for Alburquerque granitic pluton are taken from Bea (1996) (monazite analysis 2 from Table 7 and Kfs analysis 2 from Table 4). Data for Peña Negra migmatite are taken from melanosome ms-1 of Bea et al. (1994). Note the progressive lowering of partition coefficients with increasing metamorphic conditions. 
Fig. 14 als shows the estimated REE partition coefficients between monazite and K-feldspar in the lower crustal xen liths. It suggests a significant change in the behaviour of REE between both minerals as a consequence of their progressive chemical change with increasing metamorphic grade. LREE in feldspars become progressively more c»mpatible whereas monazite reduces its activity in controlling REE gechemistry. The reduced activity - f accessory phases in controlling trace element gechemistry in lower crustal scenariøs cannot be related to their disselution in the granitic melts generated during the different partial melting events. Their decrease might be related to their partial consumption during participation in dehydration (melting) reactions. As an example, if we use Eq. (3) of Spear and Kohn (1996) to model changes in trace element concentrations in a K-feldspar when monazite is being consumed, we obtain that with just a $0.0005 \mathrm{~m} \bullet 1 \%$ of consumption of monazite (supposing $\mathrm{D}^{\text {LREE }}$ monazite-K-feldspar around 30,000 from Tables 3 and 6; whereas a value of 107,000 is -btained from data of Bea et al., 1994) K-feldspar could increase their LREE contents by 15 to 50 times. This is a difference of the same magnitude as we btain when comparing LREE contents in feldspars from granulite terranes to those of granulitic xenoliths (Table 6).

\subsection{Transfers of REE, Th, $L, P$ and $Z r$ among} granulitic minerals

Crystallization of monazite and zircon during prøgrade metamorphism is usually related to the presence of pre-existing accessory minerals (Bingen et al., 1996; Pan, 1997). This is supported not only by textural relationships among accessory minerals but alse by the immobile behaviour of many trace elements during metamorphism that would require their transport •ver short distances. Formation of monazite and zircon from the breakdown or recrystallization of pre-existing REE-P-Th-U-Zr-rich trace minerals requires only very localized remobilization of these trace elements and therefore is favoured (Pan, 1997). The consumption and modal decrease of accessory minerals with increasing metamorphic grade in the studied crustal section is in agreement with this statement. Nevertheless, tw॰ situations could be envisaged in our study. In migmatite terranes, the growth and recrystallization of monazite, xenotime and zircon is mainly controlled by the previous accessory assemblage of the rocks (amphibelite to granulite facies transition related to the beginning of the biotite dehydration melting reaction). In lower crustal xenoliths, the exhaustion of accessory minerals is s- extensive that the majør silicate minerals receive a significant portion of the REE and $\mathrm{Zr}$ liberate by their breakdown (metamorphic conditions close to total consumption of biotite, in fact an Al-Ti-rich phlogepite in compsition, Villaseca et al., 1999).

In the absence of other REE-rich accessory minerals (e.g. allanite, titanite), recrystallization of monazite in peraluminous granulites is usually ascribe to apatite breakdown as demonstrate both in experiments (Wolf and London, 1995) and in detailed mineral studies (Pan, 1997; Simpsøn et al., 2000). The positive correlation between the modal decrease of apatite and the major introduction of $\mathrm{Ca}$ and $U$ in monazite (substitutions (1) and (2) above) suggests a key role of apatite in transferring not -nly $\mathrm{Ca}, \mathrm{U},(\mathrm{Th})$ but alsø $\mathrm{P}$ and REE to monazite (Fig. 15). The same situation occurs in xenotime,

REE, $Y, T h, U, P$ transfers around $1^{8 t} B t$-out isograd (migmatite terranes)

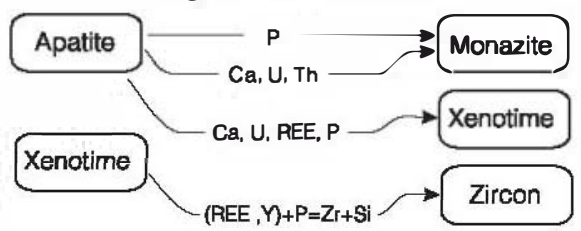

REE, $Y, Z r, P$ transfers around $2^{\text {nd }}$ Bt-out isograd (lower crustal xenoliths)

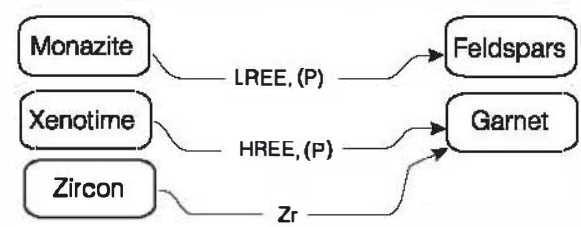

Fig. 15. Schematic wansfers of REE, Th, U, P among minerals of migmatites from granulite terranes (breakdown of biotite or first biotite-out isograd) and granulites from xenolith suite (close to Tiphlogopite-out isograd or second biotite-out isograd). 
which als• shows a marked increased in $\mathrm{Ca}$ and $\mathrm{U}$ in its crystal cores (Fig. 7). In both cases, apatite could be involved coupled with the general biotite breakdown reaction:

$$
\begin{aligned}
& \text { (U, TH, REE })+ \text { biotite }+ \text { plagioclase }+ \text { sillimanite } \\
& \quad \text { in-apatite } \\
& + \text { quartz } \rightarrow(\text { Ca, Th, U, LREE }) \\
& \quad \text { in-monazite-core } \\
& +(\mathrm{Ca}, \mathrm{U}, \mathrm{Th}, \mathrm{Y}, \mathrm{HREE})+\mathrm{K} \text {-f eldspar }+ \text { gamet } \\
& \quad \text { in-xenotime-core } \\
& \text { /cordierite }+ \text { melt }
\end{aligned}
$$

The chemical control of monazite when hosted in gamet or cordierite suggests the involvement of these minerals in some trace element transfer.

In the case of zircon, the presence $\bullet \mathrm{P}-\mathrm{Y}$-HREErich cores suggests the involvement of a phosphate in their formation and/or recrystallization. The contents of $\mathrm{Y}, \mathrm{Ca}$ and $\mathrm{P}$ in these granulitic zircons are greater than those in granitic rocks (Hoskin et al., 2000). A pløt of $Y+R E E$ (atom) vs. P (atom) reveals that for most granulitic zircons there is a $(\mathrm{REE}, \mathrm{Y}) / \mathrm{P}$ substitution of $1: 1$ (Fig. 16) suggesting that xenotime is clearly involved in their genesis transferring some HREE-Y-P components (Fig. 15).

The main røle of accessøry minerals in contrølling $\mathrm{REE}-\mathrm{Y}-\mathrm{Th}-\mathrm{U}-\mathrm{Zr}$ gechemistry of migmatite granulites changes drastically in lower crustal scenarios where accessories are progressively consumed in metamorphic reactions. The total consumption of xenotime, as manifested by its absence in the xen- liths and the high Y (and Zr)-HREE-contents in restitic garnet, suggests the involvement of these accessories (xenøtime, zircon) in their formation:

$$
\begin{aligned}
& \underset{\text { xenotime }}{(\mathrm{Y}, \mathrm{HREE}) \mathrm{PO}_{4}}+\underset{\text { zircon }}{(\mathrm{HREE}) \mathrm{ZrSiO}_{4}}+\mathrm{AlTi-phl \bullet gopite} \\
& +\underset{\text { plagiøclase }}{\text { plillimanite }}+\text { quartz } \\
& \rightarrow \underset{\text { in-garn et }}{(\mathrm{Y}, \mathrm{HREE}, \mathrm{Zr})+\mathrm{K} \text {-feldspar }+ \text { melt }}
\end{aligned}
$$

Other minerals in the xenoliths (e.g. sillimanite, quartz $\bullet$ r rutile) do n॰t sh॰w appreciable contents of these trace elements. REE-P-rich feldspars (plagioclase and $\mathrm{K}$-feldspar) are related to monazite and probably apatite breakdown, as their modal decrease in these granulites is very noticeable (Table 1). A

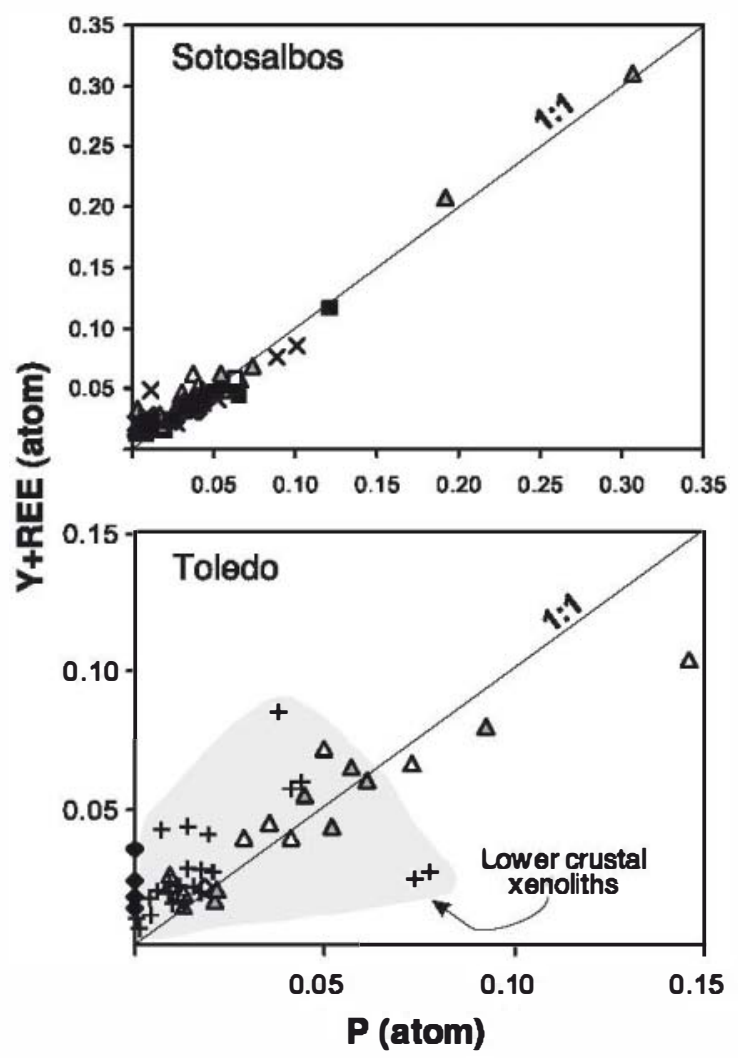

Fig. 16. Plot of Y+REE vs. P of zircons from studied samples. Zircons from lower crustal xenoliths (crosses) are included in the shaded freld. A one-to-one ratio of REE to $\mathbf{P}$ indicates xenotime substitution. All elements are in at.\%. Symbols as in Fig. 5.

general mica consumption reaction with participation -f accessories would be as follows:

$$
\begin{aligned}
& \underset{\text { monazite }}{\text { LREE) }} \mathbf{O}_{4}+\text { apatite }+ \text { Al-Ti-phløgøpite } \\
& + \text { plagiøclase }+ \text { sillimanite }+ \text { quartz } \\
& \quad \rightarrow \text { ( LREE, Na, K)A1Si } \mathbf{O}_{8}+\text { garnet }+ \text { rutile }+ \text { melt } \\
& \quad \text { K-feldspar }
\end{aligned}
$$

the residual plagiøclase being als• enriched in LREE components (Fig. 15).

\section{Conclusions}

Althøugh accessøry minerals may $\bullet c c u r$ as newly crystallized phases, $\bullet$ vergrowths and re-growths during high-grade metamorphism, there is a marked decrease of their modal abundance in lower crustal xenoliths under more extreme granulite facies conditions. 
The existence of crystal coarsening, the growth of idiomorphic and sømetimes complex oscillatory zoning (e.g. A-type zircons or type-II monazites), and the appearance of new chemical features in monazite and xenotime crystals (zircon remains more constant), lead to the supposition that most accessories in melt-rich migmatites are newly crystallized, in agreement with the data of Nemchin et al. (2001). Therefore, a redistribution of trace elements in the early stages of granulite facies conditions, during biotite-breakdown (first biotite-øut isøgrad), is required. At this stage, most trace elements are hoste in accessories although in most granulitic rocks they are isolated and interstitial, therefore separated by hundreds of microns (much more in the case of accessory-pøor lithøløgies: $\mathrm{N}$ -

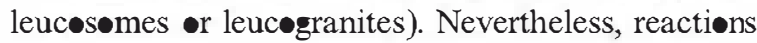
and chemical transfer between accessory minerals seem to be supported by their chemical variation and substitutions. In some melt-rich migmatites, the presence of low-HREE-Y and high-U-Ca monazite cores (or high-U-Ca xenotime cores) suggests their growth from a bighly REE undersaturated granitic melt forcing complex substitutions in accessories to stabilize them. Major minerals (feldspars, gamet, cordierite, biotite) in migmatites from granulite terranes do not show important concentrations of those trace elements but they can locally control the chemistry of the included accessory mineral (e.g. monazite in gamet or cordierite) involving the participation of accessories in major-phase reactions. Thus, there is a redistribution - f trace elements (REE, Y, Th, U, P) between accessories and major minerals as well as granitic melts in the early stages of granulite-facies conditions.

This situation changes in lower crustal scenarios where major minerals have very high contents of trace elements thus becoming the main host of the wholerock (REE-Y - $(\mathrm{Zr})$ ) budget. This $\bullet$ ccurs when Al-Tiphlogøpite is very scarce, close to total mica consumption during granulite-facies metamorphism (last biotite-øut isøgrad). A marked redistribution of HREE-Y-Zr between garnet and xenotime (until its disappearance) and zircon, but als of LREE between feldspars (K-feldspar and plagioclase) and monazite, is suggested. An intimate relationship between major and accessory phases during metamorphic reactions is deduced at high-grade granulite facies conditions as suggested recently by Pyle and Spear (1999). These •bservations have important implications in application of partitioning-based mineral-mineral and melt-mineral equilibria, which will be explored in future studies.

\section{Acknowledgements}

The authors thank the CAI-Micrøscopía electrónica de la UCM and A. Fernández Larios and J. González Tánage for SEM and electrøn micrøprøbe facilities. Hilary Downes is warmly thanked for her detailed and constructive comments. Detailed revisions made by J. Pyle, F. Bea and R. Rudnick greatly improved the quality of the work. This work has been supported by the Spanish Interministry Commission for Science and Technøløgy, prøject BTE2000-0575. [RR]

\section{Appendix A. LA-ICP-MS analyses of ATHO-G reference sample}

\begin{tabular}{|c|c|c|c|c|c|c|}
\hline & \multicolumn{6}{|l|}{ ATH॰-G } \\
\hline & $\begin{array}{l}\text { NIST } 612 \\
\text { Calibration } \\
(\mathrm{ppm})\end{array}$ & $\begin{array}{l}\text { Measured } \\
\text { (ppm), } \\
n=7\end{array}$ & S.D. & $\begin{array}{l}\text { Coefficient } \\
\text { variation }\end{array}$ & $\begin{array}{l}\text { Reference } \\
(\mathrm{ppm})\end{array}$ & $(\%$ diff $)$ \\
\hline $\mathrm{V}$ & 43.9 & 3.0 & 0.2 & 6.7 & $(4.4)$ & -31.8 \\
\hline $\mathrm{Cr}$ & 27.0 & 4.9 & 1.2 & 24.5 & 6 & -18.3 \\
\hline $\mathrm{Ni}$ & 51.4 & 3.6 & 0.7 & 19.4 & (17) & -78.8 \\
\hline $\mathbf{R b}$ & 34.1 & 52.9 & 1.4 & 2.7 & 63.8 & -17.1 \\
\hline $\mathrm{Sr}$ & 81.8 & 79.1 & 2.4 & 3.0 & 96.4 & -17.9 \\
\hline $\mathrm{Y}$ & 39.6 & 82.8 & 1.7 & 2.1 & 93.8 & -11.7 \\
\hline $\mathrm{Zr}$ & 35.5 & 606.3 & 14.6 & 2.4 & 524 & +15.7 \\
\hline $\mathbf{B a}$ & $41 . \bullet$ & 476.7 & 17.2 & 3.6 & 553 & -13.8 \\
\hline $\mathrm{La}$ & 38.6 & 50.7 & 1.2 & 2.4 & 55.5 & -8.6 \\
\hline $\mathrm{Ce}$ & 42.3 & 111.5 & 3.1 & 2.8 & 124 & -10.1 \\
\hline $\mathrm{Nd}$ & 39.8 & 55.7 & 1.9 & 3.4 & 61.3 & -9.2 \\
\hline $\mathrm{Sm}$ & 37.9 & 13.2 & 0.8 & 6.1 & 14.6 & -9.4 \\
\hline $\mathrm{Eu}$ & 37.4 & 2.5 & 0.2 & 8.0 & 2.84 & -12.2 \\
\hline Gd & 41.3 & 13.5 & 0.8 & 5.9 & 15.5 & -13.1 \\
\hline $\mathbf{D y}$ & 39.8 & 15.1 & 1.1 & 7.3 & 15.6 & -3.5 \\
\hline $\mathrm{Yo}$ & 42.0 & 10.6 & 0.7 & 6.6 & 10.1 & +4.9 \\
\hline $\mathrm{Lu}$ & 40.6 & 1.5 & $\bullet .1$ & 6.7 & 1.52 & -3.5 \\
\hline $\mathrm{Hf}$ & 38.4 & 12.2 & 0.8 & 6.6 & 13.6 & -10.1 \\
\hline $\mathrm{Pb}$ & 40.3 & 6.4 & 0.5 & 7.8 & 5.7 & +11.8 \\
\hline Th & 39.4 & 8.8 & 0.6 & 6.8 & 7.48 & +17.6 \\
\hline $\mathrm{U}$ & 39.0 & 2.5 & 0.3 & 12.0 & 2.35 & +5. \\
\hline
\end{tabular}

Reference concen ration values for ATH -G (Iceland rhyolite glass) are from Jochum et al. (2000) except ( ) which are only information values (Jochum et al., 2000).

( $\%$ diff.) is the percent deviation between measure and expected values. 
Appendix B. Granulite bulk rock geochemistry

\begin{tabular}{|c|c|c|c|c|c|c|c|c|c|c|c|c|c|}
\hline & \multicolumn{6}{|c|}{ Sotosalbos Complex } & \multicolumn{5}{|c|}{ Toledo Complex } & \multicolumn{2}{|c|}{ Xenoliths } \\
\hline & $\begin{array}{l}\text { Av. } \\
\text { orthogneiss }\end{array}$ & $\begin{array}{l}100563 \\
\text { Sotosalbos } \\
\text { granite }\end{array}$ & $\begin{array}{l}102181 \\
\text { N-leucosome }\end{array}$ & $\begin{array}{l}102183 \\
\text { Leucogranite }\end{array}$ & $\begin{array}{l}101637^{\mathrm{a}} \\
\text { Melanosome }\end{array}$ & $\begin{array}{l}100562^{a} \\
\text { Bt-rich } \\
\text { enclave }\end{array}$ & $\begin{array}{l}89103^{\mathbf{b}} \\
\text { Layos } \\
\text { granite }\end{array}$ & $\begin{array}{l}9321^{\mathbf{b}} \\
\text { N-Leucosome }\end{array}$ & $\begin{array}{l}93197^{\mathrm{b}} \\
\text { E-Leucosome }\end{array}$ & $\begin{array}{l}\$ 1925 \\
\text { Cervatos } \\
\text { leucogranite }\end{array}$ & $\begin{array}{l}93198^{\mathrm{b}} \\
\text { Melanosome }\end{array}$ & $\begin{array}{l}77750^{\circ} \\
\text { Pelitic } \\
\text { xenolith }\end{array}$ & $\begin{array}{l}99185 \\
\text { Felsic } \\
\text { xenolith }\end{array}$ \\
\hline $\mathrm{Si}_{2}$ & 66.15 & 68.62 & 74.43 & 71.81 & 60.96 & 43.19 & 61.37 & 68.92 & 69.50 & 73.49 & 57.15 & 55.02 & 64.80 \\
\hline $\mathrm{Ti}_{2}$ & $\bullet .61$ & $\bullet .51$ & 0.03 & 0.14 & 0.8 & 1.81 & $\bullet .61$ & $\bullet .13$ & 0.52 & 0.04 & 1.13 & 1.28 & 1.86 \\
\hline $\mathrm{Al}_{2} \mathrm{O}_{3}$ & 16.47 & 15.78 & 13.95 & 15.32 & 18.53 & 27.65 & 18.45 & 16.91 & 14.96 & $14.4 \bullet$ & 20.16 & 24.72 & 14.95 \\
\hline $\mathrm{Fe}_{2} \mathrm{O}_{3}$ & 5.19 & 4.11 & $\bullet .62$ & 1.10 & 8.12 & 13.57 & 7.11 & 1.56 & 2.56 & 1.2 & 9.39 & 9.94 & 8.73 \\
\hline $\mathrm{Mn}$ & 0.05 & 0.04 & $\bullet .11$ & 0.02 & 0.08 & 0.07 & 0.09 & $\bullet .01$ & 0.01 & $\bullet .02$ & 0.08 & 0.06 & 0.10 \\
\hline $\mathrm{Mg}$ & 1.67 & 1.37 & $\bullet .13$ & 0.23 & 2.58 & 3.44 & 2.68 & 0.48 & 0.91 & 0.36 & 3.39 & 2.29 & 3.22 \\
\hline $\mathrm{Ca}$ & 1.61 & 1.31 & 0.59 & 0.80 & 1.66 & 0.65 & 0.81 & 1.31 & 0.86 & 1.20 & 1.54 & - .41 & 1.40 \\
\hline $\mathrm{Na}_{2}$ & 2.88 & 2.95 & 2.45 & 3.14 & 2.34 & 1.38 & 2.01 & 2.75 & 1.83 & 3.23 & 1.79 & $\bullet .63$ & 1.87 \\
\hline $\mathrm{K}_{2}$ & 3.68 & 3.88 & 6.92 & 6.51 & 3.1 & 6.54 & 4.05 & 6.5 & 7.00 & 4.56 & 3.90 & 3.42 & 2.27 \\
\hline $\mathbf{P}_{2} \mathbf{a}_{5}$ & 0.30 & 0.26 & $\bullet .16$ & $\bullet .13$ & 0.5 & 0.1 & $\bullet .23$ & 0.3 & 0.34 & 0.21 & 0.13 & $\bullet .13$ & - .13 \\
\hline LOI & 1.35 & 1.11 & 0.56 & 0.56 & 1.58 & 1.56 & 2.20 & 0.76 & 1.16 & 1.04 & 1.06 & 1.76 & 0.78 \\
\hline Total & 99.94 & 99.94 & 99.85 & 9.76 & 100.25 & 99.96 & 99.00 & 99.62 & 99.64 & 99.75 & 99.72 & 99.66 & 100.11 \\
\hline $\mathrm{Ba}$ & 969 & 613 & 858 & 828 & 191 & 536 & 604 & 1341 & 1174 & 714 & 762 & 929 & 906 \\
\hline $\mathbf{R b}$ & 153 & 177 & 197 & 211 & 279 & 501 & 161 & 236 & 220 & 81 & 175 & 108 & 56 \\
\hline $\mathrm{Sr}$ & 173 & 148 & 137 & 213 & 88 & 79 & 173 & 276 & 247 & 182 & 237 & 141 & 248 \\
\hline $\mathrm{Y}$ & 47.10 & 33.10 & 7.12 & 41.00 & 66 & 24 & 23 & 11 & 8 & 4.15 & 27 & 46 & 52.6 \\
\hline $\mathrm{Zr}$ & 269 & 186 & 17 & 39 & 296 & 406 & 123 & 48 & 166 & 55 & 218 & 247 & 2018 \\
\hline $\mathrm{Nb}$ & 10.17 & 10.21 & 1.58 & 4.35 & 16 & 39 & 13 & 5 & 9 & 0.83 & 21 & 21 & 26.9 \\
\hline Th & 20.14 & 12.23 & 1.47 & 5.18 & 25 & 19 & 16 & 5 & 22 & 2.71 & 19 & 22 & 2.42 \\
\hline $\mathrm{U}$ & 4.33 & 7.10 & 1.11 & 5.57 & 5 & 6 & 5 & $\mathrm{nd}$ & $\mathrm{nd}$ & 1.07 & nd & nd & 1.09 \\
\hline V & 69 & 59 & 6 & 5 & 107 & 268 & 111 & 14 & 37 & 5 & 139 & 156 & 172 \\
\hline $\mathrm{Cr}$ & 290 & 256 & 76 & 20 & 607 & 249 & 364 & 6 & 25 & 164 & 145 & 246 & 119 \\
\hline $\mathrm{Ni}$ & 33 & 26 & 3 & 3 & 51 & 99 & 65 & 5 & 10 & 13 & 57 & 36 & 29 \\
\hline $\mathrm{La}$ & 51.86 & 28.43 & 3.67 & 13.10 & 53.7 & $6 \bullet .51$ & 33.55 & 23.15 & 50.98 & 8.59 & 69.41 & 68.14 & 35.50 \\
\hline $\mathrm{Ce}$ & 105.55 & 59.27 & 7.11 & 26.90 & 113.03 & 129.81 & 64.50 & 43.79 & 114.57 & 16.30 & 135.75 & 130.90 & 61.50 \\
\hline Nd & 46.09 & 29.50 & 3.24 & 11.10 & 58.4 & 65.45 & 29.13 & 21.5 & 51.31 & 6.38 & 54.80 & 63.95 & 23.50 \\
\hline $\mathrm{Sm}$ & 10.13 & 5.88 & 0.81 & 2.88 & 13.18 & 12.87 & 6.53 & 5.34 & 11.87 & 1.06 & 11.05 & 11.80 & 5.65 \\
\hline Eu & 1.67 & 1.22 & 1.13 & 1.62 & 0.85 & 1.21 & 1.25 & 2.21 & 2.06 & 1.10 & 1.93 & 2.24 & 1.96 \\
\hline $\mathrm{G} \mathbf{d}$ & 8.35 & 4.97 & 0.92 & 3.56 & 12.12 & 8.63 & 5.43 & 4.62 & 9.65 & 0.81 & 9.03 & 9.05 & 8.33 \\
\hline Dy & 7.64 & 5.14 & 1.02 & 5.00 & 11.86 & 5.28 & 4.54 & 3.36 & 3.95 & 0.69 & 7.07 & 7.59 & 7.88 \\
\hline Er & 4.67 & 2.94 & •.61 & 3.44 & 5.48 & 2.27 & 3.05 & 1.5 & 1.14 & 0.46 & 3.70 & 4.45 & 5.32 \\
\hline $\mathrm{Yb}$ & 4.88 & 2.96 & •.61 & 3.85 & 5.47 & 2.1 & 4.25 & 1.27 & $\bullet .51$ & 0.77 & 3.30 & 4.33 & 4.99 \\
\hline $\mathrm{Lu}$ & 0.81 & 0.43 & 0.09 & 0.50 & ๑. 71 & 0.35 & 0.82 & 0.23 & 0.13 & •.13 & 0.59 & 0.60 & 0.86 \\
\hline
\end{tabular}

\footnotetext{
${ }^{a}$ Data from Villaseca et al. (2001).

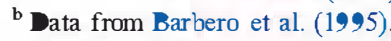

${ }^{\mathrm{c}}$ Data from Villaseca et al. (1999). 


\section{References}

Barbero, L., 1995. Granulite-facies metamorphism in the Anatectic Complex of Toledo, Spain: late Hercynian tectonic evolution by crustal extension. J. Geol. Soc. (Lond.) 152, 365-382.

Barbero, L., Rogers, G., 1999. Inplications of U-P b monazite ages from syn-orogenic granites of the Anatectic Complex of Toledo (Spain) in the evolution of the central part of the Hercynian Iberian Belt. Doc. BRGM 290, 203.

Barbero, L., Villaseca, C., 2000. Eclogite facies relics in metabasites from the Sierra de Guadarrama (Spanish Cenwal System): $\boldsymbol{P}-T$ estimations and implications for the Hercynian evolution. Mineral. Mag. 64, 815-836.

Barbero, L., Villaseca, C., Rogers, G., Brown, P.E., 1995. Geochemical and isotopic disequilibrium in crustal melting: an insight from anatectic granitoids from Toledo, Spain. J. Geophys. Res. 100B8, 15745-15765.

Bea, F., 1996. Residence of REE, $Y$, Th and $U$ in granites and crustal protoliths: implications for the chemistry of crustal melts J. Petrol. 37, 521-532.

Bea, F., Montero, P., 1999. Behaviour of accessory phases and redistribution of $\mathrm{Zr}, \mathrm{REE}, \mathrm{Y}$, Th, and $\mathrm{U}$ during metamorphism and partial melting of metapelites in the lower crust: an example from the Kinzigite Formation of Ivrea, Verbano, NW Italy. Geochim. Cosmochim. Acta 63, 1133-1153.

Bea, F., Pereira, M.D., Stroh, A., 1994. Mineral/leucosome raceelement partitioning in a peraluminous migmatite (a laser ablation-ICP-MS study). Chem. Geol. 117, 291-312.

Bea, F., Montero, P., Garuti, G., Zacharini, F., 1997. Pressure-dependence of rare earth element distribution in amphibolite- and granulite-grade gamets. Geostand. Newsl. 21, 253-270.

Bea, F., Montero, P., Molina, J.F., 1999. Mafic precursors, peraluminous granitoids, and late lamprophyres in the Avila Batholith: a model for the generation of variscan batholiths in Iberia J. Geol. 107, 399-419

Bingen, B., Demaiffe, D., Hertogen, J., 1996. Redistribution of rare earth elements, thorium, and uranium over accessory minerals in the course of amphibolite to granulite facies metamorphism: the role of apatite and monazite in orthogneisses from southwestem Nonway. Geochim. Cosmochim. Acta 60, 1341-1354.

Escuder Viruete, J., Hemáiz, P.P., Valverde-Vaquero, P., Rodriguez, R., Dunning, G., 1998. Variscan syncollisional extension in the Iberian Massif: suctural, metamorphic and geochronological evidence from the Somosierra sector of the Sierra de Guadarrama (Central Iberian Zone, Spain). Tectonophysics 290, 87-109.

Franz, G., Andrehs, G., Rhede, D., 1996. Crystal chemisty of monazite and xenotime from Saxothuringian-Moldanubian metapelites, NE Bavaria, Germany. Eur. J. Mineral. \&, $109-118$

Fraser, G., Ellis, D., Eggins, S., 1997. Zirconium abundance in granulite-facies minerals, with implications for zircon geochronology in high-grade rocks. Geology 25, 607-610.

Gerdes, A., Wömer, G., Henk, A., 2000. Post-collisional granite generation and HT-LP metamorphism by radiogenic heating: the Variscan South Bohemian Batholith. J. Soc. Lond. 157, $577-587$
Gromet, L.P., Silver, L.T., 1983. Rare earth element distribution among minerals in a granodiorite and their perogenetic implications. Geochim. Cosmochim. Acta 47, 925-939.

Hanchar, J.M., Rudnick, R.L., 1995. Revealing hidden smuctures: the application of cathodoluminescence and back-scattered elecron imaging to dating zircons from lower-crustal xenoliths. Lithos 36, 289-303.

Harris, N.B.W., Gravestock, P., Inger, S., 1992. Ion-microprobe determinations of race-element concentrations in garnets from anatectic assemblages. Chem. Geol. 100, 41-49.

Heinrich, W., Andrhes, G., Franz, G., 1997. Monazite-xenotime miscibility gap thermomey: I. An empirical calibration. J. Metamorph. Geol. 15, 3-16.

Hoskin, P.W. Ireland, T.R., 2000. Rare element chemistry of zircon and its use as a provenance indicator. Geology 28, $627-630$.

Hoskin, P.W. ., Kinny, P.D., Wyborn, D., Chappell, B.W., 2000 Identifying accessory mineral saturation during differentiation in granitoid magmas: an integrated approach. J. Petrol. 41, 1365-1396.

Jarosewich, E.J., Boamer, L.A., 1991. Rare-earth element reference samples for electron microprobe analysis. Geostand. Newsl. 15, 397-399.

Jochum, K.P., Dingwell, D.B., Rocholl, A., Stoll, B., Hofman, A.W., Becker, S., Beshmen, A., Bessette, D., Dietze, H.J., Dulski, P., Erzinger, J., Hellebrand, E., Hoppe, P., Hom, I., Janssens, K., Jenner, G.A., Klein, M., McDonough, W.F., Maetz, M., Mezger, K., Münker, C., Nikogosian, I.K., Pickhardt, C., Raczek, I., Rhede, D., Seufert, H.M., Simakin, S.G., Sobolev, A.V., Spettel, B., Sraub, S., Vincze, L., Wallianos, A., Weckwerth, G., Weyer, S., Wolf, D., Zimmer, M., 2000. The preparation and preliminary characterisation of eight geological MPI-DING reference glasses for in-situ microanalysis. Geostand. Newsl. 24, 87-133.

Johannes, W., Holtz, F., Möller, P., 1995. REE distribution in some layered migmatites: constraints on their perogenesis. Lithos 35 , $139-152$

Kret2, R., 1983. Symbols for rock forming minerals. Am. Mineral. 68, 277-279.

Kretz, R., Campbell, J.L., Hoffman, E.L., Hartree, R., Teesdale, J., 1999. Approaches to equilibrium in the distribution of race elements among the principal minerals in a high-grade metamorphic terrane. J. Metamorph. Geol. 17, 41-59.

Longeritch, H.P., Jackson, S.E., Günter, D., 1996. Laser ablation inductively coupled plasma mass spectrometric ransient signal data acquisition and analyte concen ration calculation. J. Anal. At. Spectrom. 11, 899-904

Loock, G., Stosch, H.G., Seck, H.A., 1990. Granulite facies lower crustal xenoliths from the Eifel, West Germany: perological and geochemical aspects. Con rib. Mineral. Petrol. 105, 25-41.

Martín Romera, C., Villaseca, C., Barbero, L., 1999. Materiales anatécticos en el área de Sotosalbos (Segovia, Sierra de Guadarrama). Caracterización petrológica, geoquímica e isotópica (Sr, Nd). Actas II Congr. Ibérico Geoquim., Lisb., 329-332.

Montel, J.M., 1993. A model for monazite/melt equilibrium and application to the generation of granitic magmas. Chem. Geol. $110,127-146$

Nabeleck, P.I., Glascock, D., 1995. REE-depleted leucogranites, 
Black Hills, south Dakota: a consequence of disequilibrium melting of monazite-bearing schists. J. Petrol. 36, 1055-1071.

Nash, W.P., Crecraft, H.R., 1985. Partition coefficients for race element in silicic magmas. Geochim. Cosmochim. Acta 4, 2309-2322.

Nemchin, A.A., Giannini, L.M., Bodorkos, S., Oliver, N.H.S., 2001. Ostwald ripening as a possible mechanism for zircon overgrowths formation during anatexis: theoretical constraints, a numerical model, and its application to pelitic migmatites of the Tickalara Metamorphics, northwestem Aus alia. Geochim. Cosmochim. Acta 65, 2771-2788.

Otamendi, J.E., de la Rosa, J.D., Patiño Douce, A.E., Castro, A., 2002. Rayleigh fractionation of heavy rare earths and ytrium during metamorphic gamet growth. Geology 30, 159-162.

Pan, Y., 1997. Zircon- and monazite-forming metamorphic reactions at Manitouwadge, Ontario. Can. Mineral. 35, 105-118.

Pan, Y., Fleet, M.E., Longstaffe, F.J., 1999. Melt-related metasomatism in mafic granulites of the Quetico subprovince, Ontario: constraints from - $\mathrm{Sr}-\mathrm{Nd}$ isotopic and fluid inclusion data. Can. J. Earth Sci. 36, 1449-1462.

Pereira, M.D., Rodriguez Alonso, M.D., 2000. Duality of cordierite granites relate to melt-restite segregation in the Peña Negra anatectic complex, cen ral Spain. Can. Mineral. 38, 1329-1346.

Pride, C., Muecke, G.K., 1981. Rare earth element distributions among coexisting granulite facies minerals, Scourian Complex, NW Scotland. Contrib. Mineral. Petrol. 76, 463-471.

Pyle, J.M., Spear, F.S., 1999. Ytrium zoning in gamet: coupling of major and accessory phases during metamorphic reactions. Geol. Mater. Res. 1, 1-49.

Pyle, J.M., Spear, F.S., Rudnick, R.A., McDonough, W.F., 2001. Monazite-Xenotime-Garnet equilibrium in metapelites and a new monazite-garnet thermometer. J. Perol. 42, 2083-2107.

Reid, M.R., 1990. Ionprobe investigation of rare earth element diswibution and partial melting of metasedimentary granulites. In: Vielzeuf, D., Vidal, Ph. (Eds.), Granulites and Crustal Evolution. Kluwer Academic Publishing, Dordrecht (The Netherlands), pp. 507-522.

Rubbatto, D., 2002. Zircon wace element geochemis partitioning with gamet and the link between $\mathrm{U}-\mathrm{Pb}$ ages and metamorphism. Chem. Geol. 184, 123-138.

Rubbatto, D., Williams, I.S., Buick, I.S., 2001. Zircon and monazite response to prograde metamorphism in the Reynolds Range, cen Australia. Con Mineral. Perol. 14 458-468.

Schaltegger, U., Fanning, C.M., Günther, D., Maurin, J.C., Schulmann, K., Gebauer, D., 1999. Growth, annealing and recrystallization of zircon and preservation of monazite in high-grade metamorphism: conventional and in-situ $\mathrm{U}-\mathrm{Pb}$ isotope, cathodoluminiscence and microchemical evidence. Con Mib. Mineral. Petrol. 134, 186-201.

Schwandt, C.S., Papike, J.J., Shearer, C.K., 1996. Trace element zoning in pelitic garnet of the Black Hills, South Dakota. Am. Mineral. 81, 1195-1207.
Solar, G.S., Brown, M., 2001. Petrogenesis of migmatites in Maine, USA: possible source of peraluminous leucogranite in plutons? J. Perol. 42, 789-823.

Spear, F.K., Kolın, M.J., 1996. Trace element zoning in gamet as a monitor of crustal melting. Geology 24, 1099-1102.

Tanner, D.C., Behrmann, J.A., 1997. Study of strain and partial-melt mansfer in a banded migmatite. J. Struct. Geol. 19, 14 5-1417.

Villaseca, C., Herreros, V., 2000. A sustained felsic magmatic system: the Hercynian granitic batholith of the Spanish Cen ral System. Trans. R. Soc. Edinb. Earth Sci. 91, 207-219.

Villaseca, C., Barbero, L., Rogers, G., 1998. Crustal origin of Hercynian peraluminous granitic batholiths of central Spain: petrological, geochemical and isotopic ( $\mathrm{Sr}, \mathrm{Nd}$ ) constraints. Lithos 43, 55-79.

Villaseca, C., Downes, H., Pin, C., Barbero, L., 1999. Nature and composition of the lower continental crust in central Spain and the granulite-granite linkage: inferences from granulitic xenoliths. J. Petrol. 40, 1465-1496.

Villaseca, C., Martín Romera, C., Barbero, L., 2001. Melts and residua geochemis in a low-to-mid crustal section (Cenwal Spain). Phys. Chem. Earth 26, 273-280.

Wark, D.A., Miller, C.F., 1993. Accessory mineral behavior during differentiation of a granite suite: monazite, xenotime and zircon in the Sweetwater Wash pluton, southeastem Califomia, USA. Chem. Geol. 110, 49-67.

Watson, E.B., 1996. Dissolution, growth and survival of zircons during crustal fusion: kinetic principles, geological models and implications for isotopic inheritance. Trans. R. Soc. Edinb. Earth Sci. 87, 43-56.

Watt, G.R., Harley, S.L., 1993. Accessory phase controls on the geochemis of crustal melts and restites produced during water-undersaturated partial melting. Con rib. Mineral. Petrol. $114,550-566$.

Watt, G.R., Burns, I.M., Graham, G.A., 1996. Chemical characteristics of migmatites: accessory phase distribution and evidence for fast melt segregation rates. Contrib. Mineral. Petrol. 125, $100-111$.

Wolf, M.B., London, D., 1995. Incongruent dissolution of REEand Sr-rich apatite in peraluminous granitic liquids: differential apatite, monazite, and xenotime solubilities during anatexis. Am. Mineral. 80, 765-775.

Yurimoto, H., Duke, E.F., Papike, J.J., Shearer, C.K., 1990. Are discontinuous chondrite-normalized REE pattems in pegmatite granite systems the result on monazite fractionation? Geochim. Cosmochim. Acta 54, 2141-2145.

Zhu, X.K., 'Nions, R.K., 1999. Zonation of monazite in metamorphic rocks and its implications for high temperature thermochronology: a case study from the Lewisian terrain. Earth Planet. Sci. Lett. 171, 209-220. 